\title{
DOCUMENTATION
}

\section{L'exploitation rationnelle et économique des stations hydro-électriques de faible puissance à l'aide de génératrices asynchrones.}

C'est un fait bien connu que l'exploitation des centrales hydrauliques est d'autant plus onéreuse -. unitairement parlant - - que la puissance de ces stations est plus réduite. Cela s'explique parfaitement, du reste, si l'on considère que, en dehors des dépenses de lubrifiant, d'entretien journalier et aussi dans une cerlaine mesure ... de réparations, qui, seules, sont en mapport direct avee la puissance des machines, la plus grande partie des frais d'exploitation est représentée par les salaires d'un personnel parfaitement apte à conduire une machinerie beatucoup plus puissante et, la plupart du temus aussi, suffisant pour assurer celte conduite.

Le coté faible de ces petites exploitalions élant, ainsi, surabondamment décelé ... el depuis longtemps déjà ... nombreux soml les techniciens qui se sont altachés a la recherche d'un remède pratique. Il semble bien, touldois, quaucune idée bien lunineuse ne soil sorlie de ces recherches, jusqu'à ces lemps derniers, du moins, puisque la soule solution pratique qui ait -... ¿ notre commaissance - reçu quelques applications, (encore y a -t-il lieu de souligner que e'est uniquement dans des cas d'espires), consiste à grouper plusieurs stations de faible puissance 'm une seule travaillant, quand e'est possible sans trop de frais, sous une hauteur de chute voisine de la somme des hauteurs des chutes désaffectées.

Une nouvelle solution, très élégante, vient cependant d'ètre trouvée à ce difficile problème, par MI. Lefeuvre, qui, cherchant à réaliser pratiquement, à distance, la commande automatique d'une centrale de $125 \mathrm{Kw}$. et de deux petites stations de $30 \mathrm{Kw}$, el à supprimer, de ce fait, le personnel de conduite de ces stations, fut amené à assurer cette exploitalion au moyen de génératrices asynchrones. Nous en donnerons ci-après une rapide analyse, en raison de l'intérêt qu'elle présente certainement pour nombre de nos lecteurs.

\section{Principes de l'Exploitation.}

La solution adoptée par M. Lefeuvre - el dont il rendait comple, il y a quelques mois, à la "Société française des Electriciens " - a pour base de départ la propriété caractéristique du moleur asynchrone accouplé à un matériel pouvant, à certains moments, être moteur, lequel, à partir de sa vitesse de synchronisme, débile de l'énergie au fur et à mesure de l'augmentation de vitesse pour alteindre sa pleine charge à une vitesse qui est généralement supérieure de 3 à 4 - et même $5 \%$ - à celle qui correspond au synchronisme.

L'équilibre du couple résistant de la génératrice par rapport an couple moteur de la turbine détermine la limite de vitesșe, saul, évidemment, dans le cas où il existerait entre ces derniers une disproportion par trop considérable qui permettrait le décrochage de la genératrice, ainsi qu'il adviendrạit du reste pour le moleur correspondarit. Or, dans le problème que nous étudions, ce dernier cas peut être écarté à priori, car pareil décrochage est forl loin de la puissance normale des génératrices en question qui est dimensionnée par rapport à celle disponible sur l'arbre de la lurbine (1).

Dès lors apparaît tout l'intérêt de cet ingénieux système qui, une fois en service, recevant l'énergie réactive nécessaire à son excitation, et sous la senle condition que soient constantes la hauteur de la chute (nette), la tension et la fréquence, assurera le fonctionnement également conslant, de l'exploitation, sans đqu'il soit besoin d'aucun maniement de matériel de réglage électrirue.

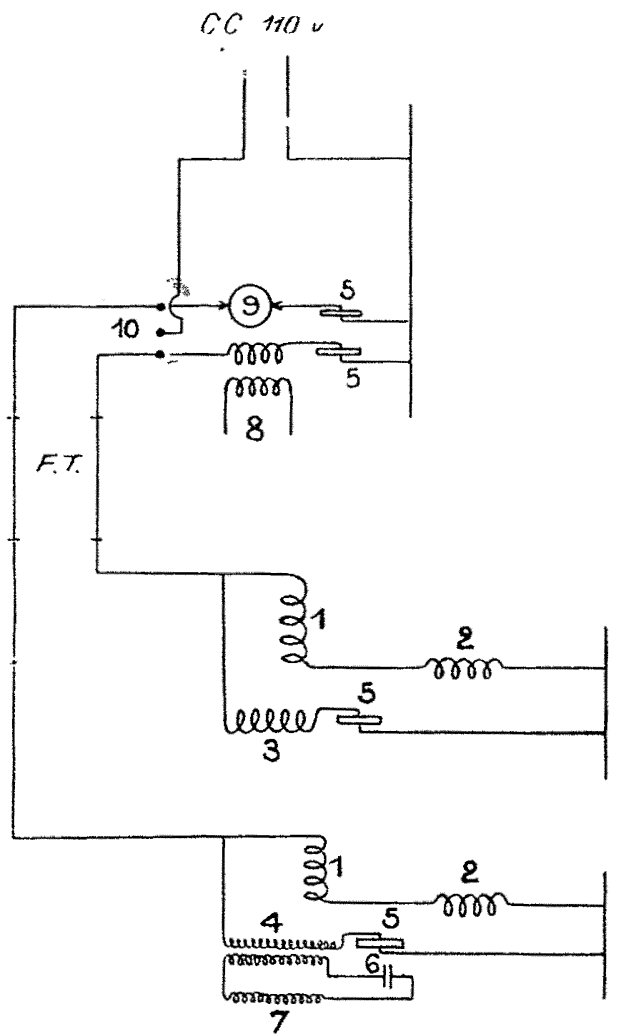

Fig. 1. - Montage schématique de la commande à distance.
(1) Relais à C. C. 110 volts avant tet arrière ;

(2) Self-inductances;

(3) Transformateur d'intensitè génératrice ;

(1) Transformateur d'intensité de niveau d'eau

(5) Condensateurs ;
(6) Lames plongeantes du niveau d'eau :

(7) Transformateur de puissance d'isolement du circuit hydraulique. (8) vattmetre-enregistreur ;

(9) Ampèremètre;

(10) Inverseur à C. C.
E.T. Fils teléphoniques.

\section{RÉAlisation PRATIQUE.}

Etant supposées réalisées, à la centrale, la tension et la fréquence constantes nécessaires, l'automaticité du fonctionnement de l'installation n'est plus qu'une question de lecture à distance des constantes de l'usine auxiliaire, de mise en route à distance

(1) Des épreuves qui durèrent plus d'un an en ont été faites avec un moteur de $25 \mathrm{C}$. V. installé sur une turbine de 35 , et dans des conditions entièrement satisfaisantes. 
de la turbine et d'accrochage de la-génératrice asynchrone lors du passage au synchronisme. Cette question a élé résolue ainsi qu'il suit, bien simplement, avec 2 des fils léléphoniques de l'ancienne installation, sans rien préjudicier au service normal du téléphone.

$1^{\circ}$ Mise en marche à distance. - La mise en marche à distance est commandée au moyen de courant continu fourni par la centrale. Ce courant est envoyé dans un relais qui met en route un contacteur spécial, lequel actionne le servo-moteur du distributeur de la turbine, dont il assure le démarrage (Fig. 1).

Ce démarrage étant acquis, lorsque la vitesse de l'engin se rapproche significativement de la vitesse de synchronisme, l'action du relais cesse et est remplacée par celle d'un régulateur centrifuge commandant un contacteur spécial, qui se subslitue alors au relais et assure l'ouverture de la turbine (Fig. 2). wattmètre qu'il excite, la puissance fournie par la station auxiliaire, et, au moyen d'un ampèremètre (qui donne des indications proportionnelles à la hauteur de plongée de 2 lames de cuivre) I'indication permanente du niveau de l'eau dans le bief.

Lorsque ce niveau varie dans des proportions appréciables, le mécanicien de garde, averti par ce dispositif de surveillance graphique, opère, par les touches du contacteur dont nous avons parlé, l'ouverture ou la fermeture partielle du distributeur de la turbine, entraînant immédialement une augmentalion ou une réduction correspondante de la puissance développée par l'engin. Enfin, si, mettant les choses au pire, l'eau devenail insuffisante, malgré cette régulation, il aurait encore la possibilité d'arrèter la marche de la station auxiliaire à partir de l'instanl où le plan d'eau dans le bief serait devenu manifestement trop déficitaire, pour la remeltre ensuite en marche lorsque la relenue normale se trouverait, de nouveau, réalisée.

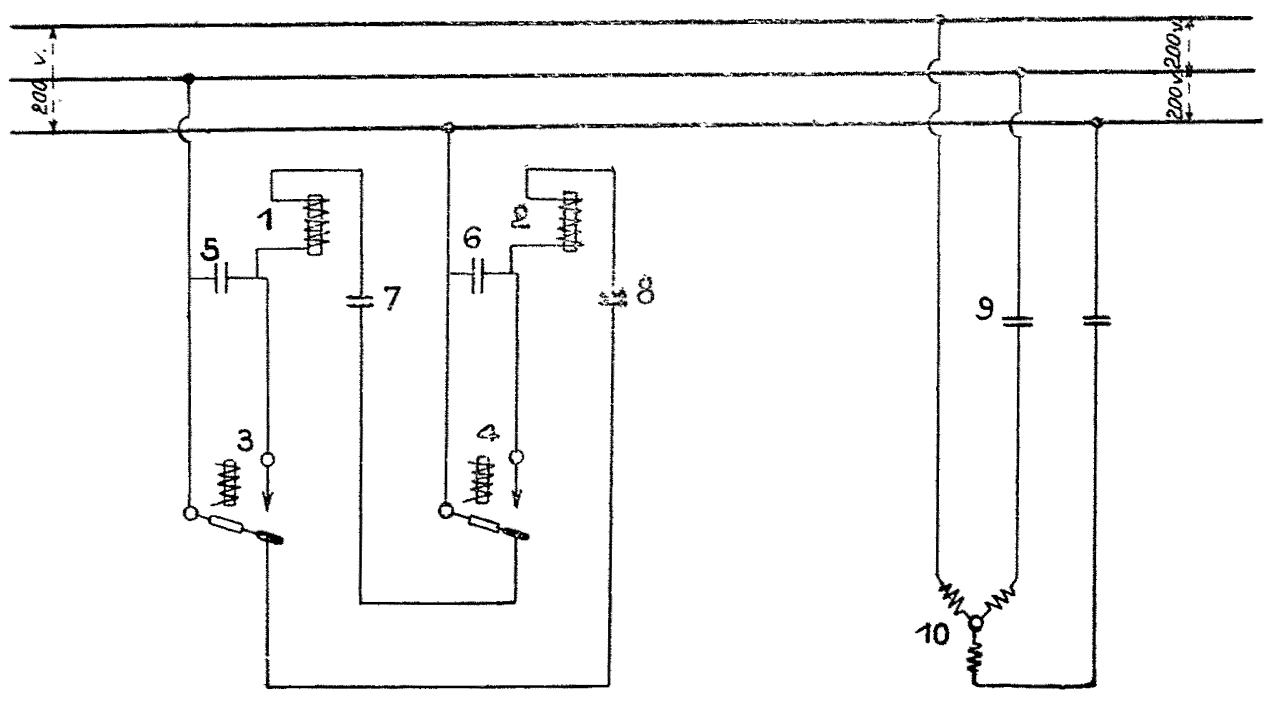

Fig. 2. - Station auxiliaire : Schéma de montage.

(1) Electro-aimant du contacteur d'ouverture des vannes ;

(2) Electro-aimant du contacteur de fermeture des vannes;

(3) Relais à C. C. (marche)

(4) Relais à C. C. (arrêt);

(5) Contacteur -régulateur (marche AV);

Contacteur-résulateur (marche $\mathrm{AR}$ )

(7) Interrupteur de fin de course (marche $A V$ )

(8) Interrupteur de fin de course (marche $\Lambda P$ )

(9) Contacteur-génératrice ;

\section{Particularités.}

Malgré sa simplicité qui lui assure une grande régularité de fonctionnemenl, la sécurité de ce système n'est pas cependant à l'abri de toute critique, et on ne les lui a pas, lout d'abord, ménagées, en particulier pour le cas où la turbine s'emballerait par suite d'un manque d'excitation des génératrices asynchrones et d'alimentation des servo-moteurs pouvant provenir d'un arrêt dans la production d'énergie de la centrale; mais le cas a été rpévu après coup.

Pour parer à cette possibilité d'incident, l'arbre de la turbine porte, calé sur lui, une poulie frein à force centrifuge, qui agit mécaniquement sur la vanne d'admission dès que la vitesse attein $15 \%$ en plus de la vitesse normale de régime et en opère la fermeture en 25 secondes environ, temps dont il suffira, évidemment, de tenir compte pour retrouver les stations auxiliaires à l'arrêt, lors de la remise sous tension des réseaux.

Enfin, d'aucuns ont fait remarquer que les lignes se trouveraient surchargées bien inutilement par l'énergie réactive que nécessite

Cette ouverture une fois assurée, aussitôt que la vitesse de la turbine arrive à être voisine de celle du synchronisme, de seulement $5 \%$ en moins, l'effet du contacteur sur le servo-moteur cessant, l'accélération de la turbine continue et, lorsque le synchronisme est réalisé, un second contacteur détermine l'accrochage de la génératrice asynchrone qui se met, dès lors, en charge progressive sur la turbine pour réaliser son fonctionnement à la puissance de régime normal quand le couple résistant de la génératrice parvient à équilibrer le couple moteur de la turbine.

$2^{\circ}$ Régime de marche et contrôle à distance. - Pour assurer la continuité du régime de marche, en particulier par la connaissance de la puissance d'utilisation et par la hauteur de l'eau dans le bief, il arrive fréquemment, en effet, que la quantité d'eau y arrivant n'est pas suffisante pour maintenir le niveau de retenue normale en amont, et il s'ensuit une diminution progressive de la vitesse qui, après avoir entraîné - par voie de conséquence directe - une réduction dans la puissance développée, peut conduire jusqu'au décrochage de la génératrice, une fois atteinte la vitesse de synchronisme.

Ce contrôle à distance permet, au moyen de dispositifs spéciaux utilisant les deux fils pilotes - et sur lesquels il serait trop long de nous élendre ici - de transmettre à l'usine, au moyen d'un l'excitation des génératrices; et cet inconvénient peut, à la vérité, devenir fort sérieux. On y obvie, néanmoins, de façon fort satisfaisante, en prévoyant, pour les machines de plus de $20 \mathrm{Kw}$, un dispositif de compensation qui permet, sur place, la production de l'énergie réactive, afin que le facteur de puissance moyen en découlant soit porté à 0,95 en avant.

Disons, toutefois, qu'il est assez malaisé de réaliser pareille compensation au moyen de génératrices synchrones synchronisées, auto-excitatrices, et qui présentent, en outre, une notoire insuffisance de glissement susceptible de présenter des inconvénients fort graves pouvant se répercuter sur le fonctionnement de l'ensemble du réseau dans le cas - toujours possible - d'une disjonction de la centrale génératrice.

$3^{\circ}$ Arrêt. - La manœuvre d'arrềt découle implicitement de ce qui vient d'être dit. Il suffit de fermer la vanne commandant l'admission; cette dernière cessant, il se produit une réduction de vitesse, et lorsque la vitesse ainsi réduite repasse par la vitesse de synchronisme, la génératrice asynchrone se déconnecte aulomatiquement.

On voit, de là, tout le profit pratique que peuvent retirer les petites exploitations hydraulicques de l'application du dispositif de M. Lefeuvre, et nous pensons que cela suffit pour nous dispenser d'insister sur son utilisalion industrielle. 


\section{La Construction de l'Usine-Barrage de Chancy-Pougny (1)}

L'aménagement de l'usine-barrage de Chancy-Pougny, sur le Rhône, fait partie du programme général d'aménagement de ce flouve, établi en 1919 par le Comité d'Etudes institué par le Ministère des Travaux Publics.

L'Usine de Chancy-Pougny est situé dans la partie du Rhône où ce fleuve forme la frontic̀re franco-suisse et entre l'usine de la Plaine, dont la construction est projetée à l'amont de ChancyPougny et l'usine des Etournelles, dont la construction est projetée l'aval.

L'jdée première de cette chute revient à un groupe franco-suisse qui s'est constitué en 1910. La Banque Suisse des Chemins de fer reprit les études commencées par ce groupe, détermina l'emplacement le plus favorable pour la construction du barrage, obtint les concessions française et suisse et les rétrocéda à la Société des Forces Motrices de Chancy-Pougny, constituée à cet effet sous l'égide des Etablissements Schneider.

Les deux concessions ont une durée de 80 ans avec droit de rachat à partir de la vingt-quatrième année.

La totalité du courant fourni est destiné à l'Energie Electrique de Thône et Jura, qui a pour client principal les usines du Creusot.

Pour l'exécution des travaux, le rôle d'Ingénieur-Conseil et d'Architecte a été dévolu à la Banque Suisse des Chemins de fer. L'entreprise générale des ouvrages de génie civil a été confiée à la Société Générale d'Entreprises, à laquelle a été associée, pour la construction du barrage, la firme Locher et $\mathrm{C}$ ie, de Zurich.

L'emplacement choisi pour le barrage est situé à $2 \mathrm{~km}$ en amont du pont de Chancy, à l'embouchure du ruisseau de Couchefatte et a été déterminé de façon à laisser au fleuve un débouché suffisant ol à fonder tous les ouvrages sur le rocher.

La chute est créée par un barrage mobile et l'usine est établie en prolongement du barage sur la rive gauche du fleuve.

Le barrage qui permet l'évacuation de crues de $1800 \mathrm{~m}^{3} / \mathrm{sec}$ comprend 4 passes de $12 \mathrm{~m}$. de longueur fermées par des vannes de $11 \mathrm{~m}$ de hauteur du type Stoney amélioré par Buss; une cincuième passe, également de $12 \mathrm{~m}$. de largeur, constitue l'écluse dont on ne construit actuellement que la tête amont. Les passes sont séparées par des piles de $2.4 \mathrm{~m}$. de hauteur, $23 \mathrm{~m}$. 50 de longueur et $3 \mathrm{~m} .50$ de largeur. Elles sont en béton armé avec libage en granit et sont relices au-dessus du niveau des plus hautes eaux par un pont en béton armé situé à l'aval des vannes.

Les treuils de manœuvre des vannes sont situés à $16 \mathrm{~m}$. au-dessus au pont sur la passerelle au-dessous de laquelle on a ménagé une galerie servant au passage des câbles électriques.

La hauteur totale de l'ouyrage, fondations comprises, atteint $51 \mathrm{~m}$.

Le seuil des passes est en béton recouvert d'un pavage en gros blocs de granit solidement ancrés dans le béton.

Le canal d'amenée est réduit à une chambre de décantation séparée du fleuve par un écran à grilles destiné à arrêter les corps flottants.

La prise d'eau de l'usine est établie dans le prolongement du barrage et comprend 5 ouvertures de $11 \mathrm{~m} .60$, alimentant chacune une des turbines principales et une petite ouverture de $5 \mathrm{~m} .60$ alimentant une turbine auxiliaire,

A l'aval des vannes de prise d'eau se trouve des grilles fines dont le nettoyage est assuré par un dispositif de retour d'eau.

L'usine est un bâtiment de $93 \mathrm{~m} .50$ de longueur sur $15 \mathrm{~m} .90$ de largeur et de $41 \mathrm{~m}$. de hauteur totale. Elle abrite cinq groupes verlicaux de $9.000 \mathrm{ch}$. et une petite turbine auxiliaire de $300 \mathrm{ch}$, Les turbines sont fournies par les Etablissements Escher Wyss et les Etablissements des Charmilles. Ce sont les plus grandes unités qui existent actuellement en Europe. Les alternateurs et l'appareillage électrique sont fournis par les Etablissements Schneider et $\mathrm{C}^{\mathrm{ie}}$.

Deux ponts-roulants de $50 \mathrm{t}$. chacun assurent les manouvres de montage de ce matériel.
Les installations établies sur la rive droite du fleuve sont relativement peu importantes et comprennent l'embranchement de chemin de fer, le poste de transformation et l'atelier de réparation.

Les alternateurs produisent le courant à la tension de $11.000 \mathrm{~V}$ et l'énergie est transportée par câbles isolés dans un bâtiment qui renferme l'appareillage.

Le courant est porté à $120.000 \mathrm{~V}$ dans les transformateurs d'un poste à ciel ouvert d'où part la ligne de transport de $160 \mathrm{~km}$ de longueur.

La chute ainsi équipée peut fournir en moyenne 160 millions de $\mathrm{kWh}$ annuels, sous une puissance qui varie de $10.000 \mathrm{ch}$. en hiver à $40.000 \mathrm{ch}$. au printemps,

Avant de commencer les travaux principaux, il a fallu résoudre des difficultés nombreuses provenant du fait de la situation spéciale du chantier situé à cheval sur la France et la Suisse, construire les maisons destinées au personnel, les voies d'accès et installer un moteur Diesel de $300 \mathrm{ch}$. destiné à alimenter le chantier en cas d'interruption du courant qui devait provenir normalement de l'usine de Bellegarde.

Les travaux principaux ont été commencés en novembre 1920 et on a procédé d'abord à l'exécution des organisations de chantier proprement dites.

Les bureaux, magasins, ateliers et places de dépôt de matériel ont été aménagés sur le plateau dominant la berge du Rhône, à droite et à gauche du ravin de Couchefatte, Ces installations sont reliées aux chantiers par deux plans inclinés et des passerelles de service. Une installation importante de fabrication de béton étagée sur une hauteur de $20 \mathrm{~m}$. a été construite et un gros matériel mécanique a été mis en œuvre pour les terrassements.

Le barrage a été entièrement construit sur caissons en utilisant deux grands ponts de service métalliques, sur chacun desquels peut se déplacer une grue portique. Les caissons en béton armé du barrage ont été construits au-dessus de l'endroit où ils devaient être immergés sur une sorte de pont-roulant s'appuyant sur les deux ponts de service.

Les caissons ainsi construits, dont certains mesuraient $24 \mathrm{~m}$. de longueur et pesaient 250 t., étaient descendus verticalement en les suspendánt à des chaînes soutenues par des vérins à vis. Lorsque les caissons des deux piles et d'un seuil d'une passe étaient foncés et que les piles étaient sorties de l'eau on exécutait, à sec, à l'abri de batardeaux, les terrassements et le bétonnage de chacun des seuils.

L'importance des terrassements exécutés a été de $300.000 \mathrm{~m}^{3}$; ceux situés au-dessus du niveau des eaux ont été effectués avec une pelle à vapeur, ceux situés au-dessous de la cote des hautes eaux ont été exécutés soit au moyen de la pelle à vapeur, soit au moyen d'un excavateur, l'épuisement étant assuré par des pompes installées sur un puisard de grande dimension placé à l'aval de l'usine.

L'organisation très soigneusement étudiée des chantiers a permis de faire tout le bétonnage des ouvrages directement des plateformes de service au moyen de coulottes. La construction en ciment armé des chambres d'eau, des volutes et des aspirateurs des turbines a été particulièrement compliquée et délicate.

Les travaux, commencés le $1^{\text {er }}$ novembre 1920 , ont été conduits exactement suivant un programme minutieusement fixé à l'avance et sont près de leur achèvement; la date de mise en eau de deux turbines est prévue pour le mois de novembre prochain.

Le succès de cette œuvre doit être considéré comme un encouragement à poursuivre progressivement le grandiose programme de mise en valeur des forces du Rhône.

(1) Extrait du procès-verbal de la séance du 23 mai 1924 de la Société des Ingénieurs civils de France.

\section{Le chauffage électrique des appartements par résistances sous les planchers.}

Particulièrement apprécié dans les pays où sont en faveur les carrelages en tomettes ou céramique (Italie, Suisse, Europe centrale et méridionale) ce nouveau système de chauffage comporte la disposition, dans l'épaisseur des planchers, des groupes de tubes parallèles, dont l'espacement est fonction du maximum de température à demander à l'installation, et qui contiennent chacun un conducteur en métal de rhéostats, isolé par un chapelct d'anneaux. Sous l'un des carreaux amovibles, une prise permet de brancher sur le secteur le nombre de tubes nécessaires pour réaliser la température désirée qui est alors fournie par la surface entière du plancher et maintenue par la masse entière de ce dernier formant volant de chaleur.

Pour être économique, ce système de chauffage nécessite toutefois l'utilisation nocturne du courant à tarif réduit et... nous n'en sommes encore point là chez nous...

Schweizerische Bauzeitung, $1^{\mathrm{er}}$ septembre. 


\section{Causes des explosions des transformateurs. - Moyens pour les éviter.}

Les explosions des appareils contenant de l'huile (transformateurs, interrupteurs à haute tension, etc.) sont dues en génćral à de l'hydrogène provenant de la décomposition de l'huile sous l'influence d'un arc. Quand on fait éclater un arc à l'intérieur d'une cuve remplie d'huile, il se dégage des gaz formés surtout d'hydrogène (environ $60 \%$ ) et d'oxyde de carbone (environ $20 \%$ ).

Pour que la grande quantité de gaz ainsi libérés puisse provoquer une explosion, il faut évidemment qu'elle trouve une quantité d'air convenable au-dessus de l'huile dans la cuve de l'appareil, et que le mélange explosif ainsi constitué soit ensuite allumé par une étincelle. Or, les limites entre lesquelles doit se tenir le pourcentage d'air dans le mélange pour que l'explosion puisse avoir lieu, sont en pratique assez étroites ; il semble qu'il faille plus de $40 \%$ d'air et moins de $80 \%$, à en croire des expériences faites avec différentes qualités d'huile. D'autre part, à moins que l'arc ne se produise juste au-dessous de la surface de l'huile, il est très improbable qu'il puisse allumer lui-même le mélange explosif, à moins que des particules incandescentes ne soient projetées avec les gaz. Tout ceci explique pourquoi les explosions de transformateurs dans l'huile sont rares.

Un appareil simple pour montrer le mécanisme de l'explosion se compose d'une éprouvette avec deux éclateurs. On noie l'un des éclateurs dans l'huile et l'on bouche l'éprouvette. En faisant éclater un petit arc à haute trension à l'intérieur de l'huile, il se dégage des gaz, et le mélange explosif formé au-dessus du liquide peut être enflammé au moyen de l'éclateur supérieur.

Il ne faut pas confondre les explosions violentes dont on vient d'expliquer la cause, avec les explosions plus fréquentes, mais beaucoup moins dangereuses, dues à la simple accumulation de gaz lors d'un arc sous l'huile et qu'une simple soupape de sûreté permet en général d'éviter.

Bien que rares, ces explosions violentes ont une telle puissance destructrice qu'on doit chercher à les prévenir. L'article montre une sous-station détruite par un tel accident lors d'un violent orage.

L'emploi d'un conservateur d'huile permet de ne pas laisser d'air dans la cuve du transformateur et, par suite, évite tout risque de formation d'un mélange explosif. Le désavantage de cette disposi- tion est que lors d'une suppression brusque provenant d'un are sous l'huile, sans formation d'un mélange explosif, il n'y a aucune couche de gaz au-dessus de l'huile pour amortir le choc ; les chances de rupture du diaphragme de sûreté ou d'un couvercle quelconque de la cuve se trouvent ainsi grandement augmentées.

La Westinghouse Electric and Manufacturing Co avait essaye d'employer un mélange d'huile el d'un corps liquide convenable (tétrachlorure de carbone ou d'éthylène par exemple), qui, tout en présentant les proprićtés isolantes de l'huile ordinaire, ne forme qu'une faible proportion de gaz inflammables sous l'influence de l'arc. Par malheur ces composés attaquent les isolants et forment des dépòts inadmissibles.

Finalement, on aboutit au principe du transformateur "inertaire ». Dans cet appareil, la cuve est hermétiquement fermée ; il y a une couche d'air au-dessus de l'huile, mais cet air a été au préalable débarassé de son oxygène. Un dispositif de soupapes s'ouvre quand la pression intérieure devient notablement supérieure à la pression atmosphérique, pour permettre l'évacuation d'une partie de l'atmosphère intérieure de l'appareil, ou bien, lorsque, au contraire, l'inverse se produit, l'air inspiré passant alors au préalable sur un composé chimique convenable, qui le débarrasse à la fois de son humidité et de son oxygène.

Grâce au jeu des soupapes (qui, en fait, sont réalisées par des fermetures hydrauliques appropriées), les échanges entre la cuve du transformateur et l'almosphère extérieure sont réduits au minimum, ce qui ménage le corps chargé de retenir l'oxygène. D'autre part, tous les risques d'explosion de la cuve par délonation d'un mélange de gaz, sont évités, puisqu'il n'y a plus d'oxygène au-dessus de l'huile. Enfin, la formation de boue par oxydation de l'huile au contact de l'air est également supprimée, sans pour cela que la cuve soit complètement remplie d'huile et, par suite, sujelle à céder facilement en cas d'unc brusque surpression intérieure qu'aucun volume gazeux n'amortirait.

Note. - Sur le système de tranformateur "inertaire " auquel il est fait allusion, on pourra consulter : Electric Journal, février 1923, page 53 et Electrical World, 31 mars 1923, page 747.

(Electrical World, $1^{\mathrm{er}}$ septembre 1923, pages 423-425, 4 figures).

\section{Réglage automatique d'un moteur synchrone destiné à compenser le déphasage d'un réseau.}

L'article décrit une sous-station automatique comprenant un moteur synchrone de $5.000 \mathrm{kwa}$, et destinée à maintenir la tension à l'extrémité d'une ligne de transmission, en fournissant ou en absorbant du courant magnétisant. Le régulateur d'excitation est un régulateur vibrant, muni d'un dispositif de surcharge limitant le débit de la machine. Le démarrage se fait par induction, à tension réduite, le champ inducteur étant relié à l'excitatrice. Le courant induit dans les bobines du champ au moment du démarrage, traverse l'induit de l'excitatrice et en retarde l'amorçage normal, ce qui permet de se passer d'interrupteur d'excitation. Un oscillogramme montre le détail de cette opération.

La sous-station n'est pas entièrement automatique. Il faut presser sur un bouton pour déterminer la mise en marche de la machine lorsque le moteur a atteint le synchronisme, le courant qu'il absorbe diminue et cette diminution déclanche un relais qui détermine l'application aux bornes dù moteur. de la tension normale au lieu de la tension réduite destinée à assurer le démarrage ; en même temps, le rhéostat d'excitation est court-circuité, ce qui est essentiel si l'on veut éviter un brusque appel de courant déwatté au moment du changement de tension. Ceci fait, la machine est placée sous l'influence du régulateur vibrant.

Les dispositifs de protection se divisent en deux classes : les uns n'arrêtent que temporairement la sous-station et permettent sa mise en marche une fois les conditions redevenues normales; les autres arrêtent définitivement la machine qui ne peut plus être remise en marche sans qu'on ait au préalable réenclanché un relais spécial.

Dans la première catégorie rentrent un relais de tension trop basse,un relais protecteur contre la rupture d'une phase ou l'échange de deux phases, des relais protégeant les enroulements contre un échauffement exagéré, le dispositif limitant l'action du régulateur, et un interrupteur de sécurité empêchant le démarrage si la pompe à huile ne fournit pas la pression voulue.

La seconde catégorie comprend un système de protection par des relais alimentés par des transformaleurs d'intensité connectés en opposition (protection contre les courts-circuits internes des machines), un disjoncteur à temps fonctionnant lorsque le moteur est trop long à se mettre en marche, et des thermostats déclanchant le disjoncteur si la température des transformateurs ou celle des paliers dépasse un certain maximum.

L'équipement automatique est plus sûr qu'un équipement confié à la vigilance d'un surveillant, et il a l'avantage de réduire les frais généraux de l'entreprise. J. M.

\section{Un nouveau système de suspension catenaire.}

L'accroissement des vitesses atteintes avec les automotrices de construction récente, a conduit à admettre des intensités de courant considérables atteignant jusqu'à 5.000 ampères pour $90 \mathrm{kwh}$. et dépassant de beaucoup les limites déjà atteintes en consommation normale. Dans le but d'assurer le bon fonctionnement des appareils mécaniques d'alimentation (pantographes ou trolleys) desservant des automotrices à forte consommation, la General Electric $C^{o}$ vient d'essayer avec succès, à Ferie, sur une ligne de 7 kilomètres de longueur, un nouveau système de suspension, rele- vant certes des principes déjà vieux, mais dont le doublage du fil de: trolley permet la disposition des joints en quinconces, disposition qui supprime tout " joint " au passage d'un fil sur l'autre et ssure une collection parfaite et sans à-coups. La ligne de contact est constituée par une file de pylônes distants de 100 mètres auxquels sont fixées des consoles supportant la ligne par l'intermédiaire d'un fil de cuivre relevant de la suspension et faisant en même temps l'office de feeder de la ligne à double trolley. J. B. 


\section{Les extensions du réseau et de la centrale de Leeds.}

Ces extensions comportent d'intéressantes innovations, en parrulier l'appareillage Reyrolle à haute tension, blindé, pour les mmandes. La nouvelle installation thermique comprend 2 turboIternateurs triphasés, de $12.000 \mathrm{Kva}$, alimentés par 2 groupes de haudières Babcox dont le service de chaufferie est assuré par ,rbines exclusivement - sauf pour les cendres, qui sont évacuées neumatiquement.

Les amplifications successives - qui semblent avoir été faites sans méthodes, au gré des circonstances, font qu'à l'heure actuelle les installations sont très complexes, produisant à la fois du diphasé 2.000 v., du triphasé 6.600 v., et du monophasé, respectivement distribués aux consommateurs à $340-400$ et 200 v. Les tramways assurent eux-mêmes leur transformation continue, et malgré les bizarreries susvisées, l'ensemble présente cependant une cohérence suffisante pour les facilités de l'exploitation.

Electrical Review, 11 mai 1923.

\section{Les essais de rendement des turbines de 55.000 CV de Queenstown.}

Dans cette intéressante étude, M. Acres rend compte des essais ratiqués sur les unités de $55.000 \mathrm{CV}$. équipant cette usine : la lerniere venue des exploitantes du Niagara, il expose par le menu es détails de ces expériences, parmi lesquels mérite d'être signalée 'application qui fut faite de la méthode Gibbson (pour la mesure le l'écoulement). Ces épreuves ont permis d'établir en fonction de a décharge les courbes de puissance et de rendement dont l'exanen atteste qu'à la puissance maximum correspond un rendement de $88 \%$, lequel atteint et même dépasse $90 \%$ entre les limites de 32.000 et $65.000 \mathrm{CV}$, avec, pour la puissance disponible aux bornes, un rendement global maximum de $91 \%$, d'où s'ensuit qu'il y a le plus grand intérêt à actionner la turbine aux environs du rendement maximum et à ne l'employer qu'exceptionnellement à la décharge maximum.

Power, 24 juillet 1923.

\section{Automotrices tramviaires commandées par vis sans fin.}

La commande par vis sans fin des automotrices de tramways, echerchéc depuis longtemps, en Angleterre, semble enfin rentrée lans la voie des réalisations pratiques : fort intéressante, elle pernet, en effet, une réduction sensible du poids mort, une consomnation de courant très inférieure aux motrices de type normal, mfin, un fonctionnement très silencieux. Les premierș de ces sngins qui viennent d'entrer en service à la "Hull Corp Tramways ", iortent des ateliers de l' "English Electric Co". Ils pèsent seulenent 9 tonnes pour une longueur de châssis de 9 mètres et sont actionnés par deux moteurs de $42 \mathrm{CV}$. suspendus à chaque extrémité, et commandant chacun l'essieu opposé, au moyen de vis sans fin, disposition qui permet de réduire à 90 kilogs le poids de l'essieu, en réalité celui de la vis... Les automotrices en question sont à impériale et peuvent contenir 60 personnes assises. Comparé à celui des voitures de mêmes caractéristiques du type ordinaire, l'entretien de ces nouveaux modèles présente une économie de $50 \%$ (entre consommation de courant et graissage).

Tramways and Railway World, 19

\section{Expériences de comparaison entre différents modèles de tubes de décharge pour turbines hydrauliques.}

Le type $1 / 4$ de cercle paraissant, depuis quelque temps, d'un rendement "indésirable ", on se préoccupe de lui en substituer un plus rationnel et surtout de rendement meilleur. A cet effet, " "Alden Hydraulique Laboratory "vient de faire toute une série d'expériences fort intéressantes dont l'auteur rend compte en ce mémoire, et qui ont porté sur des réductions des modèles Francis $24.000 \mathrm{CV}$ de "Mitchell Dam " travaillant dans des conditions identiques, avec un contrôle rigoureux et des mesures de haute précision. Soulignant que les variations de pression dans les conduites

\section{L'installation des lignes}

L'étude de M. Usbek se rapporte à un cas particulier qui comporte maints enseignements d'ordre pratique. Il s'agit de la ligne d'alimentation des chemins de fer électriques de Silésie (région de Glatz) dont l'altitude maximum ne dépasse pourtant pas 600 mètres, mais dont maintes imperfections ou conceptions erronées se révélèrent en cours d'exploitation : éclatement d'isolateurs scellés au ciment; ruplures de fils par les tempetes; surcharges occasionnces par de fortes couches de givre, de répartition irrégulière, amenant les conducteurs à glisser entre des ancrages trop espacés - et de décharge est en rapport inverse avec le rendement, il expose que, parmi les douze nouveaux modèles de tubes expérimentés, le rendement minimum $84,75 \%$ a été fourni par un conduit limité à un élément vertical de faible hauteur, et le maximum ( $88 \%$ ) par un "type concentrique " muni d'un grand cône montant jusque en haut du tube et prévu en vue d'une réalisation de la décharge, concentriquement au rotor, le plus librement possible. J. B. Proceedings of the American Society of Civil Engineers, décembre 1923.

\section{à H. T. en montagne.}

court-circuits s'ensuivant.

Chaque cas d'imperfection est étudié en détail, avec l'indication du - ou des - remèdes y apportés. Il ressort nettement de cette ćtude, fortement documentée, que les prescriptions allemandes régissant actuellement l'établissement des lignes haute tension sont insuffisantes dès que ces lignes sont situées à plus de 500 mètres d'altitude.

J. B.

\begin{abstract}
Revue Générale d' Electricité, $1^{\mathrm{er}}$ décembre 1923.
\end{abstract}

\section{Etudes sur le refroidissement des machines électriques.}

Trop importanl pour pouvoir être analysé ici, cet important rapport traite, d'un point de vue tres objectif, un nombre imposant de problemes si complexes que pose à tous points de vue, le refroiaissement des machines électriques, el décrit les appareils qui lui ont permis d'étudier, à Piltsburg, les systèmes de refroidissement es plus divers, mais tout spécialement ceux dans lesquels l'agent refroidisseur est l'air atmosphérique, contre lequel il conclut nettement en faveur des liquides comme agent d'absorption.

Après avoir donné les lois de fonctionnement des appareils à refroidissement liquide, il donne une importante partie bibliographique sur la question.

J. B.

Journ. of the Ann. Institute of Electrical Engineers, décemb. 1923.

\section{Les turbo-alternateurs de}

Ces groupes, actuellement en construction dans les ateliers de la Westinghouse $\mathrm{C}^{\circ}$ de New-York, et dont le premier sorti vient d'être installé rćcemment, doivent, au nombre de 8 , constituer l'équipement d'une des nouvelles centrales de Brooklyn de la Société Edison. Ils constituent, pour l'heure encore, le record de la puissance, avec les caractéristicues ci-après :

Turbine: Pre inn de régime, $18,6 \mathrm{Kgs}$; surchauffe à $93^{\circ} \mathrm{C}$, sous

\subsection{KVA de Brooklyn.}

\section{5 de vide.}

Alternateur : Puissance, $62.500 \mathrm{Kwtt}$; courant produit triph. $60 \sim, 13.800 \mathrm{~V}$. transmis à 27.600 , et aussi à 13.600 pour faciliter la pose des feeders dans des rues à espace libre limité. L'installation initiale a coûté 30 millions de francs or ; et l'ensemble est estimé devoir en coûter 310 . J.B.

Elettrotecnica, 15 décembre. 


\section{L'électrification des chemins de fer mexicains.}

L'électrification de la grande transversale mexicaine entre Mexico et Vera-Cruz ( $410 \mathrm{kms})$ assumée par la $\mathrm{G}$. et $\mathrm{C}^{\circ}$, vient de recevoir un commencement d'exécution dans la section d'Orizata à Esperanza (30 Kms) choisie comme début - à cause des rampes excessives qu'elle présente et d'un trafic intensif à assurer dans cette partie de la ligne.

Le courant de traction est du continu à $3.000 \mathrm{v}$. fourni par une Centrale H. E. située à $5 \mathrm{Kms}$ d'Orizata. Les automotrices sont du type mixte pour trains de charge et de vitesse sur des rampes qui par endroits atteignent $53 \%$, assez semblables aux unités du

\section{Les derniers progrès américains}

Ils ont été réalisés sous la pression des circonstances, sous l'influence de la nécessité, chaque jour plus impérieuse, d'élever les pressions et la surchauffe, qu'il s'agisse d'engins à impulsion ou à réaction.

Parmi les récents progrès qu'a constatés l'auteur, dans la construction de différentes maisons constructrices (G. E. C, Westinghouse $\mathrm{C}^{\circ}$, A. Parsons, Allis Charmer, etc.) certains, plus particulièrement remarquables, sont devenus rapidement publics, c'est
"Chicago Milwaukee and Saint-Paul " (entr'axe rigide maximum, $2 \mathrm{~m}$. 80 ; longueur totale, $15 \mathrm{~m} .85$ ), et comprenant pour chacpe unité trois bogies articulés, chacun des essieux étant conmandé directement par un moteur 1.500-3.000 v. avec trois vilesses de course normales et une à affaiblissement de champ, disposition adoptée pour permettre de réaliser l'effort de traction maximum sans dépasser le poids maximum de 20.000 kilogs par essicu. Leur équipement comporte à la fois la récupération et le contròle multiple.

Elettrotecnica, 5. décembre 1923, d'après E. R. J.

J. 13 .

\section{en matière de turbines à vapeur.}

ainsi que la Westinghouse a établi des turbines à réaction pour $84 \mathrm{~kg} / \mathrm{cm} 2$, toumant à 1.800 t. p. m., développant $50.000 \mathrm{Kw}$, et dont la vapeur d'échappement (à $21 \mathrm{k}$. 5) alimente un groupe B. P. Enfin un nouvel article présenté par la Maison Allis de Charmer, comporte une grande amćlioration du rendement, en substituant aux jeux radiaux des jeux axiaux réduits, spécialement avantageux pour l'étage $H$. P. des engins.

J. 13.

Power, $1^{\text {er }}$ janvier 1924 .

\section{L'étude rationnelle des galeries d'amenée en charge dans les installations hydrauliques.}

La question ne semble pas avoir fait de grands progrès depuis l'accident de la galerie du Riton - malgré Ies flots d'encre qu'elle a fait couler. Aussi M. Effenberger la reprend-il dans son intégralité, y apportant le fruit de son expérience personnelle, souvent ítayce sur les théories de Bûchi (assimilation de ces ouvrages aux tubes des canons - au moins pour les calculs -). La transposant ensuite dans le domaine de la pratique, il expose en détail la question des revêtements utilisés à ce jour : béton armé, asphalte, tôle, bois, etc., etc., et en particulier celle de l'assemblage des éléments, en ce qui concerne ces deux dernières sortes de revêtement.

Traitant enfin de la forme à donner aux galeries, il démontre que la section la plus rationnelle est la circulaire, qui, seule, assure une régulière répartition des pressions sur tout le périmètre de la galerie.

J. B.

Zeitschrift des Desterr. Ingenieure Vereines, 26 décembre 192\}.

\section{Règles pratiques d'entretien des transformateurs à huile.}

Fruit de nombreuses observations et déductions d'ordre pratique, cette étude résume un certain nombre de règles fort utiles à observer pour l'entretien de ces appareils - entretien généralement trop négligé - et parmi lesquelles, il y a lieu de citer :

$1^{\circ}$ La mesure - au moins mensuelle - de la résistance diélectrique de l'huile, qu'il y a lieu de maintenir à sa valeur normale par purifications régulières ;

$2^{\circ} \mathrm{La}$ réduction de l'ouverture à clapet reversible et l'interposition d'une couche d'absorbant (chaux vive) poưr éviter l'entrée de l'humidité de l'air ;

$3^{\circ}$ Pour les transfos refroidis, par circulation d'eau, des vérifications fréquentes de l'étanchéité des serpentins - et ce à une pression très supérieure (au moins double) de celle du régime normal - sur des prélévements d'huile toujours effectués an fond de la cuve ;

$4^{\circ}$ Détartrage fréquent de ces serpentins, autant.que possible à l'acide azotique ou chloryhdrique - toujours suivi d'un essal d'étanchéité ;

$5^{\circ}$ La visile annuelle du bohinage et du noyau, pour l'enlèvement des boues au jet d'huile.

Elle se termine par l'observation d'une quasi nécessité de posséder, sur toute installation un peu importante, au moins deux réservoirs d'huile : pour l'huile propre et pour celle déjà usagée.

Power, 2 octobre 1923.

J. B.

\section{Système ( collecteur " pour l'utilisation unique, dans les mêmes turbines, de l'eau de plusieurs réservoirs, ou lacs, d'altitudes différentes.}

Reprenant, en somme, le problème déjà résolu au lac Sorgniot (chute de Fully) et en élargissant le cadre, pour traiter une question d'ordre général, l'auteur démontre que, dans un système "collecteur " bien conçu, Ia conduite maîtresse partant du lac supérieur pour aboutir aux turbines, et recevant en cours de route les conduites des autres lacs, chaque prise d'eau de ces derniers doit être munie d'un clapet automatique empêchant tout reflux d'eau, et chaque confluent des conduites d'une vanne automatique à commande hydraulique dont l'ouverture puisse être déterminée par la seule pression émanant du lac le plus voisin et la fermeture être automatique dès que la vitesse de l'eau atteint un minimum préétabli, comme cela a lieu infailliblement dans le cas de rupture de conduites.

En outre, un dispositif spécial doit pouvoir permettre le remplissage progressif de la conduite forcée, el un système de ventouses, l'évacuation de l'air en découlant.

J. B.

\section{Les nouvelles applications de l'électricité à l'agriculture : Le séchage électrique des fourrages.}

Des expériences très concluantes viennent d'être tentées en Ecosse, pays très pluvieux, où le séchage des pins est trop souvent défectueux, de ce chef, et dans lequel - sous la pression des circonstances climatériques - on s'ingénie, depuis quelque temps, à trouver des procédés mécaniques permettant de l'assurer tout au moins très rapidement, sinon en complète indépendance des fluctuactions barométriques.

Le séchage électrique, expérimenté, consiste à mettre le fourrage en tas, comme avec les procédés ordinaires, mais en ménageant à l'intérieur des tas un vide mis en communication avec la conduite de refoulement d'un ventilateur dont l'action assure le séchage des herbes de l'intéricur vers l'extérieur. Ce ventilaterr n'agit du reste que par intermiltence et son action est dirigíe par l'observation de thermomètres disposés dans la masse de manière à maintenir, autant que possible, constant et dans des limites fixées d'avance, l'échaulfement que produit la fermentation.

Le prix de revient de l'opération est de beaucoup inf'érieur à celui du séchage par manutention à la main : il ne dépasse pas 0 fr. 40 la tonne (à raison d'une consommation unitaire de $1,3 \mathrm{kwh}$ ). Enfin, constatation fort encourageante, les produits sont plus nutritifs que ceux obtenus par procérlés habituels.

J. B.

Eletlrotecnica, 5 septembre 1923. 


\section{Les câbles en aluminium dans les grands transports d'énergie.}

l'économie bien connue, qui résulte de la substitution de l'aluminium au cuivre dans la constitulion des grandes lignes de force a été un des principaux facteurs déterminant du développement consiclérable cu'a pris - de date récente - l'emploi de l'aluminium en ces travaux; mais il en est aussi un autre, découlant du lait que le diamètre des conducteurs de ce métal étant plus grand - à conductibilité égale - que celui des câbles en cuivre, la tension crilique est aussi plus grande. Ėnfin, un troisième, également non négligeable, réside dans la plus grande longueur des porțées possibles.

Le calcul des lignes en aluminium est détaillé par l'auteur, qui expose également les résultats d'une étude comparațive entre les câbles en aluminium et ceux en aluminium-acier, et de laquelle résulte qu'aux prix actuels de l'acier, ces derniers sont nettement plus chers que les autres : une baisse de plus de $40 \%$ dans les cours de l'acier pourrait, seule, les mettre en etat de concurrence.

Revue Générale de l’Electricité, 3 novembre 1923.

\section{Essais de comportement des puits-déversoirs à Davis-Bridge.}

Un de ces ouvrages très spéciaux ayant été prévu pour détourner la Deerfield-River dans un tunnel de dérivation pendant la construction du barrage de Davis-Bridge (actuellement en cours), des essais de comportement furent effectués sur une maquette au $1 / 36^{e}$. Ils permirent d'établir que ce comportement confirme généralement les prévisions bascées sur la conception et le dimensionnement quand les ouvrages sont exécutés dans de bonnes conditions, et que, dans ces conditions optíma, la réduction de capacité due à l'entraînement d'air est insignifiante, aussi bien que celle inhérente à l'existence d'un vide quelconque dans la section de convergence et qu'en outre l'écoulement du déversoir est grandement facilité par l'établissement sur sa crête de guideaux convergents, spécialement si le canal d'amenée est peu profond en correspondance d'une largeur réduite.

J. B.

Proceedings of the Am. Soc. of Civil Engineers, décembre 1923.

\section{Société pour le Développement des Véhicules électriques.}

Cette Société anonyme, de constitution récente, a pour objet l'ítude, l'exploitation de tous moyens susceptibles de faciliter l'cmploi des véhicules à accumulateurs électriques, la diffusion de ces véhicules. Le siège eșt à Paris, 57, rue Pierre-Charron. Le capilal est de $1.400 .000 \mathrm{fr}$. en actions de $500 \mathrm{fr}$. toutes souscrites en numéraire. Il pourra, dès à présent, être porté à 4 millions de francs.

Le conseil d'administration est composé des sociétés et compagnies suivantes : Union d'Electricité, Ouest-Lumière, Gaz de Lyon,

\section{Les nouvelles Automotrices à courant continu}

Ces nouvelles (unités de construction suisse pour la partie mécanique, anglaise poû́r l'équipement électrique) sont destinées à assurer l'exploitation de la grande ligne, récemment électrifiée, de P'ietermaritzburg à Glencœ (275 kilom.).

Elles sont alimentées par pantographe en courant continu à 3.000 volts et peuvent, avec une puissance unihoraire de 1.200 C.V., atteindre des vitesses graduées en triple traction depuis

\section{Les derniers Trains}

D'après la description, fort documentée, qu'en donne l'auteur de cette étude, le dernier train de cette catégorie vient d'être mis en service tout récemment par la "Brooklyn-Manhattan Transit corporation "sur la ligne de la Kalle Avenue.

Il comporte deux voitures standard, longues d'environ 20 mètres et capables de 70 places, réunies par un "vestibule " relié aux chàssis par attelages à courroies et situć au-dessus du bogie mé-
Lorraine d'Electricité, Parisienne de Distribution d'Electricité, Electricité de Paris, Lyonnaise des Eaux et de l'Eclairage, le Triphasé et de MM. Henri Cahen, administrateur-délégué de l'Quest-Lumière et administrateur-directeur de la Société d'Applications industrielles; Pierre Barriș, administrateur-délégué du Sud-Lumière ; Paul Eschwege, administrateur-délégué de la Société d'Eclairage et de Force par l'Electricité à Paris ; Roger Gasquet, ingénieur ; André Mariage, administrateur-délégué de la Société des Transports en Commun de la Région parisienne.

\subsection{0 volts des Chemins de Fer Sud:Africains.}

35 jusqu'à $70 \mathrm{kms} / \mathrm{h}$. pour trains de 1.600 tonnes en rampes de 10 p. mille.

Leur chcssis, symétrique, avec cabine de commande à chaque extrémité, comporte 4 essieux. commandés chacun par un moteur de $300 \mathrm{C}$. V. avec train d'engrenages. En ordre de marche elles atteignent le poids de 67 tonnes.

J. B.

Schweizerische Bauzeitung, 8 mars.

\section{Duplex 》 de Brooklyn.}

dian. Le châssis commun est couronné d'une section de truck normal en forme de patin renversé sur lesquels glissent les ferrures amovibles des caisses, permettant une inscription parfaite dans les courbes les plus serrées.

L'équipement électrique comporte 4 moteurs avec leurs controllers. Le tonuage général atteint 36 tonnes. J. B.

Electrical Railway Journal, 11 février.

\section{Abaques pour le calcul des constantes caractéristiques des lignes aériennes à haute tension.}

Dans le calcul de l'impédance caractéristique et de la constante de propagation, l'emploi des abaques hyperboliques nécessite des calculs préalables assez longs et compliqués. Une note récente de $M$. Blondel indique une nouvelle méthode permettant l'ćtablissement de nouveaux abaques donnant directement la valeur de ces constantes en fonction des coordonnées constructives de la ligne - qu'il prend polyphasée, pour traiter la question dans son sens le plus général. Ces abaques sont certainement destinés à rendre les plus grands services dans le calcul des lignes aériennes et, comme tels, méritent d'être signalés à tous les techniciens. J. B.

Compte rendu Académie des Sciences, séance du 7 avril 1924.

\section{La Centrale municipale}

Cetle station (complètement indépendante des installations industrielles, à cause de sa destination spéciale à l'éclairage de la ville) vient de subir d'importantes modifications de modernisation. C'est ainsi que les moteurs à gaz, devenus insuffisants, ont été remplacés par deux moteurs Diesel, l'un de $520 \mathrm{C}$. V. (164 tpm) accouplé à une génératrice de $335 \mathrm{Kw}$; l'autre de $700 \mathrm{C}$. V. actionnant, de même une génératrice de $475 \mathrm{Kw}$; et que fut installé un séparateur d'huile centrifuge, qui réduit considérablement la consommation de lubriliant, en même temps qu'un P. R. de 10 tonnes d'huile.

\section{de South-River (N.-J.).}

Les prix de revient (dont l'auteur domme la statistique de 6 mois) sont particulic̀rement intéressants, et bien que, pendant cel te période, le moteur ait été soumis fréquemment à plus de $20 \%$ de surcharge, les dépenses ne ressortent, en moyenne, qu'à 9 dollars $30 \mathrm{c}$. pour $1.000 \mathrm{Kwh}$, dont :

5,75 d'huile combustible ;

0,91 d'huile de graissage ;

2,52 de frais de surveillance,

et 0,12 de dépenses diverses d'entretien.

Power, 25 décembre 1923. 


\section{Les nouvelles Automotrices mixtes Salon-Restaurant de l'Interstate Public Service $\mathbf{C}^{\circ}$.}

Ces nouvelles voitures viennent d'être mises en service récemment entre Louisville et Indianapolis (U. S. A.) et présentent un certain nombre d'imnovations fort heureuses. Leurs dimensions de caisse sont de $2 \mathrm{~m}$. 80 de largeur sur 19 mìtres de longueur ; leur poids, tout équipées, de 45 tonnes. La cabine motrice est située à une extrénité, occupant environ le $1 / 3$ de la longueur - concurremment avec la cuisine et les $\mathrm{W}$. C. - et comportant les organes et appareils de contròle des 2 moteurs de 150 C. V. actionnant l'essieu av. avec démultiplication permettant d'atteindre la vitesse de $110 \mathrm{~km} / \mathrm{h}$.
Les deux autres tiers de la voiture constituent un vagon-salon de 26 places' entre les sièges (pivolants) duquel on peut intercaler des tables démontables. Ce salon est aéré par 3 grands ventilateurs et la cuisine par un plus petit; et une installation de chaulfage électrique complète, celle de chauffage à eau chaucle, utilisée surtout pendant les grands froids.

En outre de leur marche indépendante, ces voitures peuvent servir à constituer des convois à plusicurs unités. Leur prix en usine atteignit 35.000 clollars.

Electrical Railway Journal, 22 décembre 1923.

\section{L'Electrification de la ligne de l'Arlberg.}

Cette ligne présente, comme on sait, des déclivités très fortes atteignant jusqu'à 32 p. mille. Son électrification s'imposait pour faire face à un trafic toujours croissant et l'on y travaille fébrilement sur les deux rampes. Une première section de $73 \mathrm{~km}$. vient d'être livrée à l'exploitation, en attendant l'achèvement des 63 kilomètres restant. Le courant provient des usines de Ruetz et du Spullersee qui le livrent à $55.000 \mathrm{v}$, il est distribué sous forme de monophasé 15.000 v. $16 \%$ \% Les locos déjà en service ou prévues pour y être mises lors de l'achèvement sont de 5 types comprenant en tout 53 unités, dont 7 machines d'express (2 moteurs de 500 C. V.; 6 essieux moteurs couplés par 3 , capables de $65 \mathrm{kmh}$;
20 locos pour service mixte ( 2 moteurs de 500 C. V. ; un seul sroupe de 3 essieux couplés attaqués par faux essieux et couromne dentée, capables de 70-80 Kmh. ; 20 locos-marchandises (3 moteurs de $450 \mathrm{C}$. V. attaquant les 3 essieux médians d'un couplage de 5 essieux), vitesse maximum : $50 \mathrm{Kmh}$; 4 machines d'express à 4 essieux moteurs commandés índividuellement qu'encadrent 2 bissels, vitesse maximum, $85 \mathrm{Kmh}$; enfin 2 locos d'essais a 4 et 5 essieux moteurs avec système monotriphasé, vilesses maxima 70 et $100 \mathrm{Kmh}$.

Zeitsch. des Ver. deutscher Ing., 8 mars.

\section{L'Interconnexion des Réseaux électriques en Amérique.}

Dans cette intéressante relation, l'auteur expose qu'est près d'intervenir entre les réseaux l'entente nécessaire pour réaliser l'interconnexion si désirable, et il développe tous les avantages que présentera cette dernière, de même que ses conditions de réalisation, en s'appuyant sur le rapport de la dernière convention de l' "Empire State Gaz and Electric Association" de Lake Placid (S octobre 1923).

En dehors du Niagara et du Saint-Laurent, les installations hydrauliques participeraient à cette opération pour une production qui, en 1923, atteint 1.250 .000 .000 de Kwh. et permettraient

\section{L'Installation hydraulique de}

L'aménagement de cette partie du cours de l'Hudson, où le fleuve sort des montagnes, vient d'être réalisé par l'International Paper $\mathrm{C}^{\circ}$, dans l'Etat de New-York, pour être incorporée dans le vaste réseau de l' "Adirondack Power and Light Corp ". Les conditions dans lesquelles fut créée cette installation (40.000 C. V.), décrites par les auteurs de ces intéressantes notes, fort bien illustrées, posèrent aux techniciens de toutes spécialités qui eurent à en connaître, une longue série de difficiles problèmes : à commencer par la nature très défectueuse du terrain dans la partie aménagée mi-partie granitique, mi-partie sableuse (moraines, sables et graviers mouvants très fins au bord du fleuve), qui néces- de réformer plusieurs installations vétustes, à faible rendement produisant péniblement 100.000 .000 de Kwh., en même temps que d'aménager 250.000 .000 de Kwh. supplémentaires el d'économiser près de 5.000.000 de dollars dans les achats de charbons. La plus grave difficulté qui gène encore la réalisation de cette interconnexion est l'hétérogénéité des lignes des divers réseaux, équipées toutes par des tensions spéciales variant de 66.000 à 110.000 volts. C'est, semble-t-il, ce dernier voltage qui a le plus de chances d'être admis pour le grand réseau.

Electrical World, 17 novembre 1923.

\section{L'Usinage spécial d'un Stator de $11 \mathrm{~m}$. 25 de diamètre.}

On se représente assez mal l'usinage d'une pièce pareille. Il vient pourtant d'être entrepris - et réussi - par les ateliers de la Dominion Engineering Works $\mathrm{C}^{\circ}$, de Montréal, qui, bien que spécialement outillée pour l'usinage de grosses pièces, pouvant atteindre 5,50 de hauteur et 10,50 de diamètre (aléseuse déjà tout à fait exceptionnelle...) ne possédaient point d'engins idoines pour ce travail plus que spécial. Un montage non moins spécial que décrit l'auteur de ce mémoire, permit de tourner la difficulté de la façon suivante : D'une part, le stator, en 4 pièces, fut assujetti sur 12 blocs de béton bien dressés, et, d'autre part, sur le plateau d'une aléseuse Niles (avec $\varphi=4,80$ ) 2 bancs de tour furent fixés, qui portaient, montés au diamètre intérieur, un portealésoir et un porte-outil à surfacer, tournant assez lenteinent. Une fraiseuse transportable assurait ensuite le mortaisage des queues d'aronde : le stator préalablement placé sur des piliers métalliques portant, à leur sommet, des rouleaux assurant les déplacements du stator, par roulement circulaire, pour l'amener devant l'outil à tracer (fixe).

\section{Les nouvelles Locomotives à courant alternatif de la ligne Luleö-Norvick.}

L'auteur expose d'abord les caractéristiques générales des automotrices affectées avant la guerre (1914) au service messageries, du type $1 \mathrm{C}+\mathrm{C} 1$, pesant 138 tonnes et capables - avec 2 moteurs - d'assurer en double traction la remorque de convois de 2.100 tonnes (dont 1.400 utiles à la vitesse de $50 \mathrm{Km} / \mathrm{h}$. en palier, et, en simple traction, celle de convois de 1.200 tonnes (dont $1.050 \mathrm{nt}$.) à $30 \mathrm{kms}$, en rampes de $10 \mathrm{p}$. mille. Les résultats obtenus ont été satisfaisants au point que la direction de la Compagnic vient de mettre en service (1922-23) 11 unités nouvelles du même modèle, mais plus puissantes ( 4 moteurs de choc, $700 \mathrm{C}$. V. attaquant par pignon une grande couronne dentéc commandant les bielles).

Comme particularités dignes de mention, signalons que le réglage des tensions à partir du transfo, de chaque demi-loco, differe de celui des anciennes locomotives. Longues de $20 \mathrm{~m}$. 90, et pesant $127 \mathrm{t}$., elles sont capables d'un effort au crochel de $28.000 \mathrm{kgs}$ et d'une vitesse de $60 \mathrm{~km}$ en palier (30 sur rampes de $10 \mathrm{p}$. mille) remorquant une charge de $2.000 \mathrm{t}$. Ajoutons qu'une simple transformation d'engrenages leur permettrait d'assurer, à la vitesse de $100 \mathrm{~km} / \mathrm{h}$, le service de trains rapides.

Zeitsch. des Ver. deutschen Ing., 26 janvier 1924. 
Descartes savant, par Gaston Mrimaud, Professeur à la Sorbonne. Félix Alcan, édileur. - Prix 12 fr. 50. - En vente à la Librairie de la Houille Blanche, 23, Grande-Rue, Grenoble.

I.e but que s'est donné l'auteur de cette étude, fort remarquable d'ailleurs, est à la fois scientifique et national. En effet, on a essayé de tout temps de mettre en doute la franchise et la bonne foi du grand philosophe, et cela non seulement dans les questions touchant aux controverses religicuses, questions dans lesquelles cette hypothèse semble parfois justifiée, mais, et surtout, dans les domaines complètement indépendants de toute influence et où l'honorabilité scientifique du grand penseur ne peut être suspectée.

N'a-t-on pas essayé d'attribuer la loi des sinus à Svellius, l'explication de l'arc-en-ciel à Dominis, les théorèmes relatifs à la théorie des équations à Harriot ou à Viète, etc. Evidemment, au point de vue des résultats, il importe peu que telle ou telle découverte ait ćtćfaite par l'une ou par l'autre personne. La formule de Cardon, par exemple, conserve toute sa valeur, bien que cette formule ait ćté donnée par Tartaglia, maître de Cardon.

Néanmoins, les grands services que Descartes a rendu à l'humanité et le souvenir sacré que nous gardons du grand penseur nous oblige à veiller à ce que rien ne soit enlevé de ce que lui est dù. Et c'est là le grand mérite de l'auteur de cet ouvrage qui a su, grâce à son érudition profonde, mettre au point les faits dont la haute portée nationale ne peut être contestée.

D'autre part, l'auteur ćtudie, avec une documentation rare, le développement graduel des différentes phases d'imagination de Descartes, fait une analyse de ses œuvres mathématiques et montre les conclusions auxquelles le grand philosophe devait être conduit, conclusions qui ont servi de fondement à la science cartésienne. Et c'est précisément cette partie de l'ouvrage qui doit particulièrement intéresser les ingénieurs, car c'est elle qui exerce l'esprit à ' la bonne méthode et apprend dans quel ordre les raisonnements doivent être enchaînés et déduits les uns des autres pour qu'on puisse dégager de l'ensemble une solution pratique. Un ingénieur, plus peut-être que tout autre, admire le nom de Descartes. C'est l'ingénieur qui se sert constamment de graphiques, abaques, etc. C'est lui qui est très souvent conduit à se rappeler que la base de la représentation graphique des formules, procédé qui rend celles-ci plus claires et directement applicables, est la géométrie analytique dont le créateur fut Descartes.

\section{SMoukovitch.}

\section{***}

Traité complet de T. S. F., par J. Morel. - Un volume in-16 de 308 pages, 160 figures, 3 tableaux hors-texte. - Editeur : Librairic Garnier Frères, 6, Rue des Saint-Pères, Paris. - Prix, broché, 9 ir. - En vente Librairie Rey, 23, Grande-Rue, Grenoble.

Le but de cet ouvrage de vulgarisation est d'exposer d'abord, par des notions simples, toutes physiques, et sans formules, les phénomènes de cette science nouvelle et merveilleuse qu'aucun ne doit plus ignorer, et de faire suivre cette étude de données pratiques de réalisation, àfin de la mettre à la portée de tous. L'auteur rappelle d'abord les éléments d'électrotechnique, indispensables, puis passe en revue les phénomènes d'oscillations, de couplage, de détection el d'émission d'ondes électromagnétiques.

La lampe à trois électrodes, pivot de la T. S. F. actuelle, est ensuite examinée en détail dans les rôles multiples qu'elle remplit, détection, amplification et ćmission; puis viennent ses applications aux postes récepteurs, des plus simples aux plus complexes, jusqu'aux types à super-hétérodyne et super-régénération Armstrong.

L'ouvrage se termine par des domnées pratiques de construction relatives à lous ces systèmes récepleurs, ainsi que par des renseignements très intéressants sur les organes accessoires entrant dans leur constitution, tels que : transformateurs, condensateurs, récepteurs, etc.
Enfin une annexe donne des tables pour l'établissement facile des bobines d'accord de tous genres.

Le traité de T. S. F. de M. Morel est un ouvrage essentiellement pratique s'adressant aussi bien au profane qui veut s'initier aux mystères de la T. S. F. qu'à celui qui veut essayer son habileté à la réalisation d'un poste récepteur.

(S. V.).

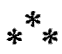

Circulaire du 19 octobre 1923 du Ministère des Travaux Publics sur Les Barrages de grande hauteur résistant par leur propre poids. - Une plaquette $16,5 \times 25$ de 48 pages, avec tableaux et figures. - Prix : 2.50. - Editeur : Librairie de l'Enseignement Technique L. Eyrolles, 3, rue Thénard, Paris $\left(V^{e}\right)$. - En vente : Librairie Rey, Grande-Rue, 23, Grenoble.

La construction des barrages-réservoirs de grande hauteur destinés à l'aménagement des chutes d'eau et à la régularisation du débit des cours d'eau, constitue l'un des problèmes importants que les ingénieurs ont à étudier.

Si, dans l'établissement de ces ouvrages, on doit avant tout considérer la sécurité, il ne faut pas oublier qu'une diminution dans les énormes dépenses de construction permet d'abaisser le prix de revient de l'énergie et par suite d'áménager certaines chutes qui seraient restées improductives.

La circulaire du Ministre de l'Agriculture, en date du 15 juin 1897 , n'a été rédigée qu'en vue de la révision et du contròle des barrages existants à cette époque, c'est-à-dire, à un moment où l'on considérait comme exceptionnelles des hauteurs qui sont couramment dépassées.

Etant donnée l'importance de présenter cette question, sous l'action du Ministre des Travaux Publics, le Conseil Général des Ponts et Chaussées a chargé une commission d'ingénieurs des Ponts et Chaussées de déterminer, dans l'état actuel de la science et de l'art de la construction, les règles à suivre pour la préparation des projets et l'exécution des travaux des barráges de grandes hauteurs. La circulaire du 19 octobre 1923, résume le rapport de cette commission, approuvée par le Conseil Général des Ponts et Chaussées, dans sa séance du 21 juin 1923.

(S. V.).

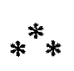

Guide du Consommateur d'Energie électrique à la Ville et à la pampagne. - Une brochure $14 \times 21$, de 64 pages, publiée par l'Union des Syndicats de l'Electricité, 25, boulevard Malesherbes, à Paris. - Prix 1 fr. 80 .

L'Union des Syndicats de l'Electricité vient de publier sous ce titre une brochure de vulgarisation dans laquelle elle donne au public, sous une forme très facilement accessible, toutes les indications nécessaires aux usagers de l'électricité.

Le texte est rédigé d'une façon à la fois très claire et très attrayante et il contient tout ce qu'un homme de notre époque doit savoir sur l'électricité, tant pour pouvoir s'en servir que pour connaître les dangers qu'elle présente.

Après un court exposé des conditions dans lesquelles l'énergie électrique est produite et transportée, la brochure passe successivement en revue tout ce qui concerne les branchements, l'isolement, les interrupteurs, les coupe-circuit, les prises de courant, les lampes, les petits moteurs, les compteurs et les tarifs de vente du courant.

Elle contient en outre un grand nombre de conseils qui seront appréciés sur l'établissement des installations intérieures, les soins qu'elles comportent et sur les conditions d'une bonne utilisation.

Cette brochure, qui a été rédigée à la demande de la Fédération Nationale des Collectivités d'Electrification rurale, est en vente à l'adresse indiquée ci-dessus. Une réduction importante est faite pour toute commande de 100 et de 1.000 exemplaires. 


\title{
INF ORMATIONS
}

\section{Union des Syndicats de l'Électricité}

\author{
25, Boulevard Malesherbes - Paris
}

\begin{abstract}
RAPPORT sur les essais d'interrupteurs horaires faits au Laboratoire Central d'Electricité et à l'Éco e d Horlogerie de Paris, établi par la 5e Cammission (chauffage électrique).
\end{abstract}

\section{Introduction}

En raison des difficultés momentanẻes qụ'éprouvent la plupart des distributeurs d'énergie électrique à fournir au moment de la pointe les quantités d'énergie qui sont nécessaires aux appareils de chauffage électrique au fur et à mesure que l'usage de ces appareils se développe, c'est la multiplication des appareils à accumulation de chaleur que l'Union est amenée à recommander en premier lieu.

L'application de ce système permettrait de consentir des tarifs réduits pour la vente du courant, à condition qu'on soit en possession d'appareils interrupteurs pouvant automatiquement couper et remettre le courant à des heures déterminées.

La question s'est donc posée de savoir si les appareils que l'industrie est actuellement en mésure de fournir présentent les garanties de bon fonctionnement et de durée nécessaires, et la $5^{\mathrm{e}} \mathrm{com}-$ misșion a été amenée à procéder à des essais de divers appareils qui ont eu lieu au Laborataire Central d'Electricité et à l'Ecole d'Horlogerie de Paris. Les appareils devaient être essayés :

$1^{\circ} \mathrm{Au}$ point de vue de leur bon fonctionenment et notamment de la bonne conservation des contacts de fermeture et d'ouverture du circuit ;

$2^{\circ} \mathrm{Au}$ point de vue de la régularité de marche et de la résistance à l’usure.

Les divers constrụcteurs d'interrupteurs ont été invités à remettre au Laboratoire des exemplaires de leurs appareils et à en vérifier eux-mêmes l'installation sur place.

Si tous n'ont pas répondu à cette invitation, le nombre d'appareils remis (21 interrupteurs et 4 relais) s'est trouvé néanmoins suffisant pour permettre une comparaison instructive.

\section{Essais effectués par le Laboratoire central d'Electricité}

Comme il était à prévoir que certains interrupteurs pourraient fournir une marche de plusieurs années, on ne pouvait songer à entreprendre l'essai pur et simple ayec la périedicité normale (c'est-à-dire quotidienne ou bi-quotidienne) de l'appareil. La commission a donc demandé aux constructeurs d'accélérer un au moins des exemplaires remis, de telle façon que les essais puissent se faire dans un délai acceptable.

Ce mode d'essai est d'ailleurs défavorable à l'interrupteur. Bien qu'avec la fréquence employée (variant de 12 secondes à 2 minutes), les contacts n'aient pas chauffé d'une façon appréciable, l'ensemble du mécanisme tournait à une vitesse pour laquelle il n'était pas prévu, et certains enroulements (tels que cẹux des remontages électriques), destinés à une marche essentiellement intermittente, ont reçu le courant d'une façon pour ainsi dire permanente.

Néanmoins, la plupart des constructeurs ont accepté de remettre des appareils accélérés.

Nous verrons plus loin que certains de ces appareils ont fourni un nombre d'interruptions correspondant à plus de dix ans de marche avec des contacts restés en très bon état. Il a donc paru que la continuation des essais jusqu'à destruction risquerait de les prolonger fort longtemps et que cette première série d'expériences fournissait un enseignement pratique déjà très suffisant pour le but que la commission avait en vue.

Dans les essais de contacts, la charge était constituée par des lampes. Pour réduire la dépense de courant, elle était coupée aussi vite que l'appareil le permettait (la durée de l'émission élait de 5 à 7 secondes).
Les appareils à remontage électrique ont fonclionné d'une façon poụr aịsi dire continue. Les appareils a remontage à main subissaient au contraire un temps d'arrêt à chaque remontage. Un voltmètre ou un ampèremètre enregistreur, intercalé dans le circuit, permettait de déterminer la période des fermetures et ruptures.

Lorsqu'un appareil pouvait fonctionner indifféremment sur continu et sur alternatif, on choisissait les conditions les plus cléfiavorables, c'est-à-dire le courant continu. Lorsqu'il existait deux exemplaires, l'un était essayé sur continu, l'autre sur alternatif.

Voici quelques détails sur les ạppareils essayés (1).

Appareils A. - Trois appareils ont été remis, dont les caractéristiques sont les suivantes :

Puissance : 110 volts, 10 ampères.

Remontage à main ;

Contact à mâchoires, unipolaire.

Deux appareils ont été accélérés et essayés, l'un sur le continu, l'autre sur l'alternatif.

Le premier de ces appareils (continu) a fourni 3.182 interruptions, c'est-à-dire l'équivalent de plus de 8 ans et demi de marche à rạison d'une interruption par jour. L'appareil est resté en état de fonctionnement, bien que ses contacts aient été détériorés par un incident d'ordre mécanique (desserrage d'un petit écrou de fixation des mâchoires, qui a donné un contact à la masse. Après resscrrage l'appareil a repris sa marche sans aucune réparation).

Le second appareil a eu son ressort cassé après 1.335 interruptions. Les contacts sont en très bon état.

Appareils B. - Deux appareils ont été remis, avec les caractérịstiques suivantes :

Puissance : 250 volts, 25 ampères ;

Remontage à main ;

Conțact unipolaire à 2 ruptures sur le mercure.

Le constructeur n'ayant pas remis d'appareil accéléré, il a été impossible de faite l'essai des contacts.

Appareils C. - Trois appareils ont été remis, donl un accéléré, avec les caractéristiques suivantes :

Remontage à main;

Contact unipolaire à 2 ruptures sur le mereure.

Le ressort s'est cassé au début et n'a pu être essayé.

Appareils D. - Un appareil accéléré a élé remis, dont les caractéristiques sont les suivantes :

Puissance : 110 volts, 30 ampères ;

Remontage à la main ;

Contact unipolaires à 2 ruplures.

L'appareil a fourni 1.380 interruptions, après quoi les contacts étaient encore bons.

Tel qu'il était au début, l'apparcil devrait avoir ses contacts visités el nettoyés au moins lous les trois mois.

Appareils E. - Deux appareils, dont un accéléré, ont été remis dont les caractéristiques sonl les suivantes :

Puissance : 110 valts, 15 ampères ;

Remontage par trembleur synchrone, commandé par la détente du ressort (ne fonctionne que sur le courant alternatif) ;

Contacts par une sorte de contrôleur bipolaire à deux ruptures par pôle.

L'apparcil accéléré a fourni 6.398 interruptions sans incident et reste en état de fonctionner.

(1) Nous désignons par une letlre chacun des construeteurs l'ordre étant quelconque. 
Apparerrs F: - Quatre appareils, que nous désignerons par les $\mathrm{n}^{0 \mathrm{~S}} \mathrm{~J}, \mathrm{II}, \mathrm{III}, \mathrm{IV}$, ont élé remis :

Deux sont à remontage électrique par disque tournant et ne foncliomnent par suite que sur le courant alternatif : le n 1 (accéléré) coupe diractement par un interrupteur à mâchoires unipolaires et à un seul contact ( 110 volts, 7,5 ampères) ; le $n^{\circ} I 1$ (triphasé) coupe par un interrupteur à mâchoires à 2 contacts par pôle ( 220 volts, $3 \times 30$ ampères).

Deux sonl à remontage à main : le $n^{\circ}$ III pour courant continu (accéléré), à coupure unipolaire par un seul contact (110 volts, 7,5 ampères) ; le $n^{\circ}$ IV (triphasć), dans lequel l'horloge n'intervient que pour mettre le courant sur un servo-moteur à disque tournant commandant l'interrupteur, qui est à deux contacts par pôle (220. volts, $3 \times 30$ ampères).

Jans l'un de ces appareils, le courant est fermé à la main; le mécanisme horaire n'intervenant que pour le déclanchement. La fermeture ne peut en conséquence avoir lieu qu'entre les heures prévues pour l'émission.

L'appareil no III a ćté essayé et a fourni 3.869 interruptions sans incident; l'appareil reste en état: de fouctionner.

Appareils G. - Six appareils, à 220 volts, dont un triphasé (3 $\times 30$ ampères) ont été remis.

Deux sont à remontage à la main, et quatre à remontage par petit moteur à collecteur, allant indifféremment sur courant continu ou sur courant alternatif.

l'es essais de rupture ont eu lieu sur trois appareils (que nous désignons pax les numéros V, VI et VII);

V. - Ln appareil à contact unipolaire à balai, remonté à la main (essayć sur courant alternatif);

VI. - Un appareil à contact unipolaire à màchoires, remonté électriquement (essayé sur alternatif);

VII: - Un appareil à. contact unipolaire à màchoires, remonté dectriquement (essayé. sur continu).

L'appareil $n^{\circ} \mathrm{V}$ a fourni 3.196 interruptions de courant à 10 ampères sans incident et reste en état de fonctionner.

L'appareil $n^{\circ}$ VI a fourni $1.7+0$ interruptions de coürant à 10 ampères après lesquelles les contacts sont restés en bon état, mais le moteur de remontage tourne sans que le ressort s'arme

L'appareil no VII. a fourni. 3.7.98 interruptions : après 2:400 interruptions, le moteur de remontage a grippé :'il'assuffi - d’ajouter un petit tampon graisseur pour que lappareil continue sans incident. E'interrupteur travaillait sur un relai, c'est-à-dire avec une trës faible intensité.

En dehors de ces interrupteurs horaires, deux constructeurs nous ont remis des relais.

Appareils H. - Un premier constructeur nous a remis un relai (110 volts, 10 ampères'), qui commandé par l'àppareil G no VII, a fourni 3.798 interruptions sans aucun incident:

Appareils I. - Un deuxième constructeur nous a remis trois modèles différents de relais :

L'un a un seul électro-aimant; une émission de courant enclanche l'appareil, l'ćmission suivante, par'le même fil'; le déclanche (ce relai n'a: pas étié essayé):

L'autre a deux électro-aimants (un pour l'enclanchement et un pour le déclanchement). L'une des bohines. de cet appareil a été mise hors service par échaulfement anormal, qui: ne: se serait vraisemblablement pas produit si lappareil avait fonctionné avec sa fréquence habituejlc.

Le troisième à enclanchement et déclanchement par le même fil, mais par deux électro-aimants différents. Cet appæreil fonctionne loujours après 3.196 interruptions.

Conclusion. - La conclusion à tirer de ces essais est qu'il existe des appareils qui rćpondent bien au but qu'on se propese, c'est-àdire copables de fermer ou d'ouvrir à des heures déterminées un circuit de 110 ou 220 volts; jusqu'à une quinzaine d'ampères, soit directement, soit par l'intermédiaire de relais, ces appareils pouvant fonotionner sans autne soin qu'un entrietien pou dispendieux, une dizaine d'années (et probablemant davantage), à raison d'une émission par jour.
A une ou deux exceptions près, les incidents survenus paraissent inhérents, non pas au système lui-même, mais à une petite imperfection de l'exemplaire essayé.

\section{Essais complémentaires}

EsSAIS DE COURT-CIRCUIT. - Le Laboratoire central d'Electricité a procédé en outre à des essais destinés à renseigner la Commission sur la résistance mécanique des appareils lorsqu'un court-circuit se produit sur le circuit qu'ils commandent.

A cet effet, les appareils étaient alimentés à la tension alternative de 110 volts (valeur efficace) et de fréquence 42, fournie par un transformateur de $10 \mathrm{k}$. V. A. Au circuit commandé par l'interrupteur était substitué un interrupteur muni d'un fusible calibré, fondant instantanément pour une intensité de 25 ampères lorsque l'interrupteur était fermé.

Tous les appareils ont été soumis à cet essai. Deux seulement dont les interrupteurs très faibles sont destinés à couper le circuit de commande de relais, ont été mis hors de service.

\section{Essais effectués par l'Ecole d'Horlogerie}

Les essais ont porté :

$1^{\circ}$ Sur la valeur du mouvement au point de vue de la régularité et des effets de changement de température (1) ;

$2^{\circ}$ Sur la construction des mécanismes d'horlogerie;

$3^{\circ}$ Sur les différents systèmes de fermeture des circuits.

Dans ce qui suit, on désigne par "écart de compensation ", l'influence de température, et par " écart de marche " la différence qui résulte, en dehors des variations de température, des diverses causes d'irrégularité (différence de tension des ressorts, frottements, etc...).

Les écarts de compensation sont donnés pour une différence de température d'enxiron 20 degrés et pour une durée de 24 heures, sauf indication contraire.

Les écarts de marche sont donnés pour des périodes indiquées dans chaque cas.

Resultats Des essais. - Voici les résultats des essais :

Appareils A. - Remontage tous les quinze jours.

Eoart de compensation; trois minutes d'avanoe.

Ecarts de marche :

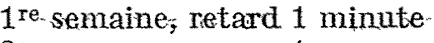

$$
\begin{aligned}
& \begin{array}{llll}
2 & - & - & 1 \\
3^{\mathrm{e}} & - & - & 0
\end{array}
\end{aligned}
$$

Appareils $B$. - Remontage tous les mois. $1^{\text {er }}$ appareil : Ecart de compensation, 2 minutes de retard. Ecarts de marche :

$$
\begin{aligned}
& 1^{\text {re }} \text { semaine, avance } 12 \text { minutes }
\end{aligned}
$$

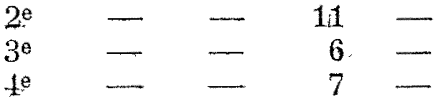

$2^{\mathrm{e}}$ appareil : Compensation bonne.

Ecarts de marche :

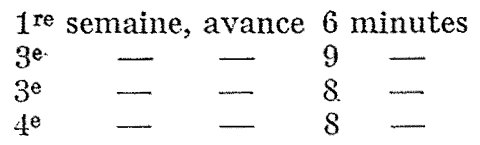

Appareils C. - Remontage tous: les quinze jours. Le ressort d'encliquetage devrait être mieux fixé.

$1^{\text {er }}$ appareil : Mauvaise compensation, retard de plus de deux minutes par jour sous l'effet de la chaleur.

Marche irrégulière :

$1^{\text {re }}$ semaine, retard 19 minutes.
$2^{\mathrm{e}}-\quad-\quad 8$
$3^{\mathrm{e}}-14-$
$4^{\mathrm{e}}-\quad-\quad 21-$

(1) Les appareils ont été soumis à des écarts de température de 20 a. $\mathrm{C}$. 
$2^{\mathrm{e}}$ appareil : Ecart de compensation, avance de 45 secondes. Ecarts de marche :

$$
\begin{aligned}
& 1 \text { re semaine, retard } 0 \text { minute } \\
& \begin{array}{llll}
2^{\mathrm{e}} & - & - & 1 \\
3^{\mathrm{e}} & - & 0 & - \\
4^{\mathrm{e}} & - & - & 1
\end{array}
\end{aligned}
$$

Appareils $F$. $-1^{\mathrm{er}}$ appareil : remontage chaque mois. Ecart de compensation, retard 2 minutes.

Ecarts de marche :

$$
\begin{aligned}
& 1^{\text {re }} \text { semaine, retard } 5 \text { minutes }
\end{aligned}
$$

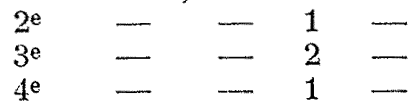

$2^{\mathrm{e}}$ appareil : remontage électrique.

Ecarts de marche :

$$
\begin{aligned}
& 1^{\text {re }} \text { semaine, retard } 7 \text { minutes } \\
& 2^{\mathrm{e}}-8 \text { - } 8 \text { - } 7 \text { - }
\end{aligned}
$$

$3^{\text {e }}$ appareil : remontage électrique.

Ecart de compensation, retard 2 minutes.

Ecarts de marche :

$$
\begin{aligned}
& 1^{\text {re }} \text { semaine, retard } 0 \text { minute } \\
& 2^{\mathrm{e}}-{ }^{1 / 2}- \\
& 3^{\mathrm{e}}-2^{\mathrm{C}}- \\
& 4^{\mathrm{e}}-{ }_{1}^{1 / 2}-
\end{aligned}
$$

Appareils $G .-1^{\mathrm{er}}$ appareil : remontage électrique, compensation assez bonne, retard inférieur à 30 secondes par jour.

Ecarts de marche :

$$
\begin{array}{lll}
1^{\text {re }} \text { semaine. retard } 3 \text { minutes } \\
2^{\mathrm{e}}-3^{1 / 2}= \\
3^{\mathrm{r}}-3^{\mathrm{r}}= \\
4^{\mathrm{e}}-3^{\mathrm{e}}= & -3
\end{array}
$$

$2^{e}$ appareil : remontage à la main, marche un mois. Compensation très bonne, écart intérieur à 20 secondes par semaine.

Ecarts de marche :

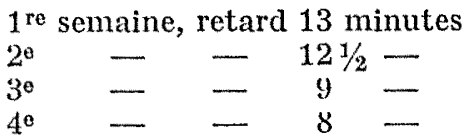

$3^{\mathrm{e}}$ appareil : remontage électrique (balancier circulaire). Compensation très bonne, écart inférieur à cinq secondes par jour.

Ecarts de marche :

$$
\begin{aligned}
& 1^{\text {re }} \text { semaine, retard } 8 \text { minutes } \\
& 2^{\mathrm{e}}-10 \quad-
\end{aligned}
$$

Conclusion. - Dans l'ensemble, les appareils sont bien conçus, robustes et capables d'assurer un travail sérieux.

Cependant, le moyen de réglage des mouvements laisse à désirer, aucun mobile à grande vitesse n'étant accessible.

La compensation est souvent défectueuse. Enfin, divers détails constructifs sont susceptibles de perfectionnements.

C'est ainsi que l'axe du balancier devrait toujours être vertical; en effet, lorsque l'axe est placé horizontalement, le défaut d'équilibrảge du balancier augmente encore le défaut d'isochronisme des. oscillations.

L'Ecole d'horlogerie a suggéré quelques modifications de détail dont les constructeurs pourront faire leur profit.

\section{Conclusion générale}

Les résultats résumés ci-dessus montrent que les constructeurs sont à même de fournir aux secteurs de distribution d'énergieélectrique des appareils interrupteurs horaires d'un fonctionnement. sûr.

Rien n'empêche donc les secteurs d'installer chez leurs abonnés. des appareils de chauffage par l'électricité à accumulation de chaleur utilisant le courant de nuit.

La certitude que l'interrupteur horaire empêchera les abonnés de mettre leurs appareils en circuit à d'autres moments que pendant les heures de nuit fixées d'avance, permet d'envisager pour l'avenir des réductions de tarifs qui rendront le chauffage électrique véritablement avantageux.

\section{Essais contrôlés de Véhicules électriques à Accumulateurs}

organisés pour septembre 1924 par l'Union des Syndicats de l'Electricité, boulevard Malesherbes, 25, à Paris, avec le concours dur Ministëre de la Guerre, de l'Office National des Recherches Scientifiques el Industrielles et des Inventions, de la Commission Technique de l'Automobile-Club de France et du Laboratoire Central d'Electricité.

\section{REGLEMENT}

Article premier. - L'Union des Syndicats de l'Electricité avec le concours du Ministère de la Guerre, de l'Office National des Recherches Scientifiques et Industrielles et des Inventions, de la Commission Technique de l'Automobile-Club de France et du Laboratoire Central d'Electricité, organise une seconde série d'essais contrôlés de véhicules électriques à accumulateurs.

Ces essais auront lieu dans la région parisienne du 15 au 26 septembre 1924 .

Arr. 2. - Catégories. - Les véhicules seront classés, suivant la charge utile qu'ils transporteront effectivement, en 7 catégories :
$1^{\text {re }}$ catégorie, type $500 \mathrm{~kg}$ : charge utile de
1 à $600 \mathrm{~kg}$;
$2^{\mathrm{e}}$ catégorie, type $1.000 \mathrm{~kg}$ : charge utile de 601 à $1.200 \mathrm{~kg}$;
$3^{\mathrm{e}}$ catégorie, type $1.500 \mathrm{~kg}$ : charge utile de 1.201 à $1.750 \mathrm{~kg} ;$
$4^{\mathrm{e}}$ catégorie, type 2 tonnes : charge utile de 1.751 à $2.500 \mathrm{~kg}$;
$5^{\text {e }}$ catégorie, type 3 tonnes : charge utile de 2.501 à $3.500 \mathrm{~kg}$;
$6^{\mathrm{e}}$ catégorie, type 4 tonnes : charge utile de 3.501 à $5.500 \mathrm{~kg}$;
$7^{\text {e }}$ catégorie, type 6 tonnes : charge utile de $5.501 \mathrm{~kg}$ et au-dessus.

Les tracteurs seront classés suivant la charge utile qu'ils peuvent transporter tant sur eux-mêmes que sur leurs remorques.

Chaque catégorie pourra se subdiviser suivant le type de l'accu- mulateur (plomb, fer-nickel, etc.) et suivant que lo parcours sera effectué avec ou sans recharge partielle de la batterie.

Chaque participant ne pourra présenter dans chaque catégorie plus de 3 véhicules.

Ant. 3. - Objet des essais. Mesures. - Les essais ont pour objet. de déterminer pour chaque véhicule :

$1^{\circ}$ L'énergie ćlectrique consommée par le véhicule pendant chaque parcours :

a) par tonne-kilomètre utile ;

a) par tonne-kilomètre tolale ;

$2^{\circ}$ L'énergie prise à la station de charge pendant la durée lotale des essais et l'énergie consommée par les voitures pendant la même période;

$3^{\circ}$ La vitesse commerciale réalisée à chaque parcours.

Ils comportent en outre l'observation de la régularité de marche.

Art. 4. - Publication des résultats. - Aucune communication relative aux essais, et en particulier aux résultats obtenus, ne pourra être faite que par l'Union des Syndicats de l'Electricité, ou avec son autorisation.

Les parlicipants pourront déclarer se retirer de l'épreuve à tout moment, même après les essais, mais au plus tard huit jours francs après l'envoi, qui leur sera fait par l'Union des Syñdicats de l'Elec- 
tricité, des résultats obtenus par eux. Dans ce cas, leurs résultats ne seront pas publiés.

Ant. 5. - Appareils de mesure. - La consommation sera mesurée par des compteurs d'énergie placés sur la voiture et sur les tableaux de charge et fournis par l'Union des Syndicats de l'Electricité. Ces appareils seront étalonnés par le Laboratoire Central d'Electricité.

Ant. 6. - Dépense d'énergie. - La dépense d'énergie électrique sera supportée par les participants.

AnT. 7. - Parc. - Les véhicules devront porter d'une manière apparente le numéro d'ordre qui leur sera indiqué, les chiffres seront peints en blanc sur fond foncé et devront avoir au moins 25 centimètres de hauteur.

Les véhicules seront garés l'avant-veille du début de l'épreuve et pendant toute la durée de cette dernière, dans un parc comportant des postes de charge pour les accumulateurs et situé à Bellevue, à l'Office National des Recherches Scientifiques et des Inventions.

Pendant l'épreuve, les véhicules seront placés sous le régime des parcs fermés.

ART. 8. - Batteries d'accumulateurs. - Les batteries portées par les véhicules devront :

$1^{\circ}$ Etre d'un des modèles courants fabriqués en vue de la vente ;

$2^{\circ}$ Avoir, sauf dérogation accordée par l'Union des Syndicats de l'Electricité, des dimensions conformes à la normalisation arrêtée par le Syndicat professionnel des Industries Electriques ;

$3^{\circ}$ Etre constituées du nombre d'éléments correspondant à la charge sous 110 volts. Pour les véhicules de $1^{\text {re }}$ catégorie, la charge pourra se faire par groupes de voitures.

Les participants auront la faculté de produire le contrat de gárantie délivré par le constructeur de leurs batteries. Les stipulations principales de ces contrats seront publiées dans le rapport qu'établira l'Union des Syndicats de l'Electricité sur les essais.

ART. 9. - Charge et recharge des accumulateurs. - Chaque participant devra faire établir lui-même les tableaux de charge de ses roitures, conformément aux indications qui lui seront données par l'Union des Syndicats de l'Electricité. Ces tableaux devront être remis au plus tard le $1^{\text {er }}$ septembre 1924 à l'Office National des Recherches Scientifiques et des Inventions à Bellevue (Seine-etOise) et seront réceptionnés par le Laboratoire Central d'Electricité.

La charge des accumulateurs sera faite par les participants sous la surveillance des commissaires préposés aux essais.

Elle ne pourra être faite qu'entre 19 heures et 7 heures.

Les recharges partielles ne pourront être faites qu'au parc et entre midi et 13 heures $1 / 2$. L'énergie prise pendant ces recharges sera mesurée.

ART. 10. - La tension du courant fournie aux heures de charge sera de 110 à 115 volts.

Les prises de courant pour la charge devront être conformes au type normalisé par le Syndicat professionnel des Industries Electriques.

Akт. 11. - Entretien et réparations. - Le conducteur du véhicule ou, à son défaut, un remplaçant, pourra seul et sans aide procéder à la visite et aux réparations mécaniques de son véhicule pendant les deux heures qui suivront la rentrée au parc.

Toute opération d'entretien des accumulateurs autre que l'entretien de l'électrolyte ne pourra se faire qu'en présence et avec l'autorisation d'un commissaire spécialement désigné qui en dressera le compte rendu. Pour assurer le contròle dans ces conditions, les batteries seront fermées el plombées, sauf les orifices nécessaires à la fuite des gaz el à l'entretien de l'électrolyte.

L'entretien et la manipulation des accumulateurs ne pourront être faits que pendant les deux heures qui suivront la rentrée au parc. Le conducteur du véhicule pourra se munir du matériel utile et se faire aider dans son travail par le persomnel nécessaire.

En cours de route, les scules réparations autorisées seront effectuées par les moyens du bord et seulement par le conducteur du véhicule.
Toute dérogation à ces prescriptions du règlement fera l'objet d'une mention spéciale dans le rapport sur l'épreuve.

ARt. 12. - Horaire. - L'épreuve sera effectuée d'après un horaire déterminé et publié avant le commencement des essais.

Le parc ne sera ouvert que de 8 à 19 heures, sauf pour le personnel préposé à la charge des accumulateurs.

A l'heure précise prévue à l'horaire, le véhicule sera considéré comme ayant pris le départ.

Le retour au parc devra être effectué au plus tard à 17 heures.

Un arrêt d'une heure et demie à Bellevue sera obligatoire pour tous les véhicules au passage du nœud de la boucle dont il est question ci-dessous.

ART. 13. - Itinéraires et prescriptions de route. - Un plan donnera les itinéraires journaliers comprenant des points caractéristiques où les commissaires du bord noteront les passages des véhicules et l'heure de ces passages.

Ces itinéraires seront en forme de 8 , le nœud de la boucle étant à Bellevue, où se feront les départs et les rentrées.

La durée totale de l'épreuve sera de dix jours et le parcours journalier à accomplir avec ou sans charge partielle pour chaque véhicule sera d'environ :

$90 \mathrm{~km}$ pour les les $1^{\mathrm{re}}$ et $2^{\mathrm{e}}$ catégories ;

$75 \quad$ - la $3^{\mathrm{e}}$ catégorie ;

$60 \quad-\quad$ les $4^{\mathrm{e}}$ et $5^{\mathrm{e}}$ catégories ;

$50-\quad$ les $6^{\mathrm{e}}$ et $7^{\mathrm{e}}$ catégories.

La vitesse des moyennes commerciales réalisées par chaque véhicule pendant toute la durée des essais ne devra pas être inférieure à:

$18 \mathrm{~km}$ à l'heure pour la $1^{\text {re }}$ catégorie ;

$15-\quad-\quad$ les $2^{\mathrm{e}}$ et $3^{\mathrm{e}}$ catégories ;

$12-\quad-\quad$ les $4^{\mathrm{e}}$ et $5^{\mathrm{e}}$ catégories;

10 - la $6^{\mathrm{e}}$ catégorie ;

8 - la $7^{\mathrm{e}}$ catégorie.

En aucun cas la vitesse maximum, en descente, des véhicules ne devra dépasser de plus de $30 \%$ la vitesse maximum en palier.

Les stipulations du Code de la route, l'arrêté du 25 janvier 1923 du Ministère des Travaux Publics et les arrêtés municipaux relatifs aux allures sur les voies parcourues devront être rigoureusement observés.

Art. 14. - Commissaires de bord. - Chaque véhicule aura à bord, outre le conducteur, un commissaire pour lequel un siège confortable et abrité de la pluie devra être aménagé. Ce commissaire qui ne devra jamais abandonner la voiture, relèvera les indications des appareils de mesure au départ, aux points caractéristiques de l'itinéraire et à l'arrivée.

Le commissaire notera sur une feuille de route, qui lui sera remise en temps utile, les heures de départ, de passage aux points caractéristiques et d'arrivée, et en général, tous les incidents de la route.

Art. 15. - Charge utile. - La charge utile pour toutes les voitures engagées dans l'épreure se composera exclusivement, outre le poids du commissaire afférent à chaque voiture et estimé à $70 \mathrm{~kg}$, de caisses de gravillons fermées et solidement arrimées sur les voitures; le poids de ces caisses sera uniformément de $35 \mathrm{~kg}$.

Ces caisses seront fournies par le participant.

Une caisse d'appoint sera autorisée s'il y a lieu.

De fréquentes vérifications du poids transporté seront effectuées.

Pour les voitures de transport de voyageurs, les voyageurs qu'elles seront déclarées susceptibles de transporter seront représentés obligatoirement par des caisses de gravillons de $35 \mathrm{~kg}$ à raison de $70 \mathrm{~kg}$ par voyageur. Ces caisses seront placées à l'endroit même où seraient les voyageurs qu'elles représentent.

Art. 16. - Pesées. - Les véhicules seront pesés à vide avant les essais et au cours de ceux-ci.

Le poids à vide comprendra le poids du châssis et de la carrosserie, celui de la batterie, celui du conducteur, le poids de l'outillage, le poids des pièces de rechange, $y$ compris les roues et bandages 
de rechange. Le poids de la charge utile sera obligatoirement compris dàn les limites spécifiées pour la catégorie daus laquelle est inscrit le véhicule et devra rester le même pendant toute la durée de l'épreuve.

Les vêhicules ayant tout d’abord été pesés à vide, le poids de la charge utile sera vérifié:par une pesée du véhicule chargé.

ArT. 17. - Pièces de rechange. - Un inventaire des pièces de rechange emportées à bord sera établi par le participant, et le commissaire de bord prendra cet inventaire en charge. Il co:tròlera T'emploi éventuel des pièces.

Les pièces avariées et remplacées prendront dans le chargement la place des pièces neuves ntilisées. Elles devront être représentées, sauf impossibilité.

A la fin de l'ẹpreuve, l'inventaire sera contrôlé.

Art. 18. - Annexes. - Des annexes détermineront ultérieurement les conditions de détail de l'épreuve.

ArT. 19. - Essais spéciaux. - En 'vue de déterminer la consommation de chaque véhicule aux différenfes vitesses, il sera procédẻ à des essaís spéciaux :en palier et en rampe qui seront subis sur un même tronȩon d'itinéraire par tous les véhicules, à différentes vitesses fixées dans chaque catégorie.

Le règlement spécial relatif à ces épreuves est annexé au présent règlement.

Art. 20. - Modifications au règlement. - L'Union des Syndicats d'Electricité se réserve le droit d'apporter au présent règlement toutes modifications qu'elle jugera utiles.

ArT. 21. - Inscription et droit d'engagement. - Les constructeurs désirant participer aux essais devront se faire inscrire à l'Union des Syndicats de l'Electricité, boulevard Malesherbes, 25, à Paris, avant le 15 juin 1924, en indiquant le nombre et la nature des véhicules qu'ils présenteront.

Des inscriptions pourront toutefois être reçues jusqu'au 15 juillet, mais le droit d'engagement sera majoré de $50 \%$ pour les engagements qui parviendront entre le 15 et le 30 juin, et de $100 \%$ pour ceux qui parviendrant entre le $1^{\text {er }}$ et le 15 juillet.

Du fait de leur inscription à l'épreuve, les participants s'engagent à observer le:présent règlement.

Un droit d'engagement sera versé par les participants au moment de leur inscription. Ce droit sera par véhicule de :

500 francs pour la $1^{\text {re }}$ catégorie.

$750---2^{\mathrm{e}}-$
$1.000-3^{\mathrm{e}}-$
$1.250-1 \mathrm{ks} 4^{\mathrm{e}}$ et $5^{\mathrm{e}}$ catégories.
$1.500-6^{\mathrm{e}}$ et $7^{\mathrm{e}}-$

Les sommes ainsi versées resteront acquises à I'Union des Syndicats d'Electricité, alors même que le "vẻhicule ne participerait pas à l'épreuve pour quelque cause que ce soit ou que le participant déclarerait se retirer de l'épreuve comme cela est prévu à l'article 4 .

Art. 22. - Responsabilités. - L'Únion des Symdicats de l'Electricité décline toutes responsabilités de quelque nature qu'elles soient, étant entendu que celles-ci incombent aux participants ayant engagé leurs véhicules.

Art. 23. - Formule d'engagement. - Dans la formule d'engagement qu'ils signeront, les participants devront spécifier :

Qu'ils acceptent en toutes parties le présent règlement;

Qu'ils dégagent les organisateurs de toutes responsabilités, de quelque nature qu'elles soient, et prennent celles-ci à leur charge ;

Qu'ils se sont assurés à une Compagnie d'assurances notoirement solvable contre tous les accidents causés soit à eux-mêmes, soit au commissaire placé à bord de leur véhicule, soit à des tiers (la police dinssurance devra être produite avant le clépart, sous. peine d'exclusion de l'épreuve);

Qu'ils déclarent accepter toutes décisions de l'Union des Syndicats de l'Electriclté concernant l'application du règlement et s'engagent à ne s'adresser en aucune circonstance aux'tribunaux.

L'engagement devra être accompagué d'un tableau de renseignements fourni par le participant et conforme à un modicle qui sera établi par l'Union des Syndicats de l'Electricité.

\section{ANNEXI POUR LES WSSAIS SPECINUX}

Article premier. - Conformément à l'article 19 du règlement des essais contròlés, et en vue de déterminer la consommalion de chaque véhicule aux différentes vitesses, les sparticiparits devront faire exécuter à leurs véhicules, dans des conditions déterminées ci-dessous, un trajet déterminé :

$1^{\circ}$ En palier;

$2^{\circ}$ En rampe.

Art. 2. - Essais en palier. - Une base en palier sera parcourue par chaque véhicule un certain nombre de fois, de telle façon que le parcours total, effectué les jours où auront lieu les essais, soit sensiblement de même longueur que litinéraire-type prévu à l'article 13 du règlement, pour la catégorie à taquelle le véhicule appartient.

Ces essais seront effectués à différentes vitesses moyennes relevées par des chronométreurs.

Ces vitesses moyennes seront celles des régimes normaux du véhicule, indiquées par le participant dans la feuille de renseignements annexée à la formule d'engegement.

Elles ne pourront être inférieures aux minima indiqués à l'article 13 du règlement pour les moyennes des vitesses commerciales.

Arr. 3. - Au cours de ces épreuves, l'énergie dépensée par lo véhicule et autant que possible le débit de la batterie seront relevés par le commissaire de bord.

ART. 4. - Essais en rampe. - Des essais en rampe se feront sur la côte de Bellevue. Ils seront effectués immédiatement avant et après les essais en palier, prévus à l'article 2 de la présente annexe.

Au départ les véhicules auront à descendre la côte de Bellevue et à la remonter immédiatement après. A la suite de cette épreuve les véhicules redescendront la côte et partiront pour les essais en palier.

Ils seront alors l'objet des mesures spécifiées à l'article 3 de la présente annexe.

Au retour de ces essais et pendant la remontée des véhicules au parc, se fera une nouvelle série de mesures.

$\mathrm{Au}$ bas et au sommet de la côte de Bellevue se trouveront un chronométreur et un commissaire du Laboratoire Central a'Electricité qui noteront respectivement :

$1^{\circ}$ Les heures de départ el d'arrivée;

$2^{\circ}$ Les indications fournies par les appareils de mesure.

\section{Le Gerant : P. LEGENDRE}

Anc. Etabts Legendre, 14, rue Bellecordière, Lyon. - J. Balaillard, Directeur. 